Research Article

\title{
A Series of Barbituric Acid Derivatives from Sulfa Drug: Synthesis and Antimicrobial Activity
}

\author{
Mahmood Muhi Fahad Ezzat Hussein HZimam, Majed Jary Mohamad \\ Department of Chemistry, Faculty of science, University of Kufa, Iraq. \\ Corresponding author. E-mail: almullaemad@gmail.com
}

Received: Jun. 9, 2018; Accepted: Feb. 5, 2019; Published: Mar 6, 2019.

Citation: Mahmood Muhi Fahad, Ezzat Hussein Zimam, and Majed Jary Mohamad, A Series of Barbituric Acid Derivatives from Sulfa Drug: Synthesis and Antimicrobial Activity. Nano Biomed. Eng., 2019, I I ( I): 67-83.

DOI: 10.5101/nbe.v11i1.p67-83.

\begin{abstract}
This paper reports the synthesis and characterization of some new barbituric acid derivatives from sulfadiazine. A reaction of sulfadiazine with chloroacetyl chloride gave 2-chloro-N-(4-(N-pyrimidin2-ylsulfamoyl) phenyl) acetamide [A] which was reacted with thiourea and $\mathrm{K}_{2} \mathrm{CO}_{3}$ to give thiazole derivative [B]. Schiff base compounds $\left[\mathrm{Sh}_{1}-\mathrm{Sh}_{3}\right]$ were prepared from condensation of thiazole derivative with different aromatic benzaldehydes. Then, addition reaction of acetyl chloride to Schiff bases afforded new tertiary amides compounds $\left[\mathrm{D}_{1}-\mathrm{D}_{3}\right]$. The latter compounds were allowed to react with 1, 3-bis (hydroxyl methyl) barbituric acids derivatives $\left[\mathrm{E}_{1}-\mathrm{E}_{2}\right]$ via Williamson reaction to form new barbituric acid derivatives $\left[F_{1}-F_{3}\right]$ and $\left[G_{1}-G_{3}\right]$. Thin layer chromatography, melting points, Fourier transform infrared spectroscopy (FTIR), proton nuclear magnetic resonance $\left({ }^{1} \mathrm{H}-\mathrm{NMR}\right)$ and carbon-13 nuclear magnetic resonance $\left({ }^{13} \mathrm{C}\right.$-NMR) techniques confirmed formation of the prepared compounds. Antimicrobial studies of the synthesized compounds were assayed against three different types of bacteria, including Staphylococcus aureus, Pseudomonas aeruginosa and Escherichia coli, and against two types of fungi Aspergillus flavus and Candida Albicans. Biological applications of the synthesized compounds showed a greater effect on antimicrobial activities than the standard.
\end{abstract}

Keywords: Sulfadiazine; Thiazole; Schiff base; Barbituric acid; Antimicrobial activity

\section{Introduction}

Sulfadiazine (4-amino-N-pyrimidin-2-ylbenzenesulfonamide) is a compound that contains two reactive locales, One of these aromatic amine and the other is sulfonamide [1]. It is a drug that belongs to the sulfonamide group and has been utilized in veterinary and human therapy over sixty years [2], and in the treatment of urinary tract infections (UTI) [3]. Thiazole is a main structure for an important class of nitrogen $(\mathrm{N})$ and sulfur (S) containing heterocycles, in particular [4], The univalent radical is known as thiazolyl. The molecule of thiazole is planar, and the C-S bond length equals $171.3 \mathrm{pm}$, similar to that in thiophene compound [5]. The applications of thiazoles were found in drug development for the treatment of hypertension, allergies, schizophrenia, inflammation, bacterial, HIV infections, and hypnotics and in treatment of pain [6].

Schiff bases are formed when any primary amine reacts with an aldehyde or a ketone in absolute alcohol with the presence of a few drops of glacial acetic acid or p-toluene sulfonic acid or concentrated hydrochloric acid [7]. These compounds also have applications in food chemistry, agro chemical, dye industry and 
pharmaceuticals [8]. Barbituric acid was prepared by Adolf Von Baeyer in 1864 from a fusion of the urea and malonic acid [9]. It is an organic compound based on a pyrimidine heterocyclic skeleton [10]. Barbituric acid is the parent compound of barbiturate drugs, although barbituric acid itself is not pharmacologically active [11], and the pharmacological properties of barbiturates mainly depend on the side groups attached to the C-5 atom of the pyrimidine ring [12]. A number of 5-alkyl- and aryl barbiturates are used as hypnotic, sedative, anticonvulsant, and antihypertensive drugs [13].

This research involved the synthesis and characterization of some new barbituric acid derivatives from sulfadiazine and the study of their antimicrobial activities.

\section{Experimental}

All reagents and solvents were purchased from commercial sources and used without purification. Melting points were recorded using electro thermal melting point apparatus. Fourier transform infrared spectroscopy (FTIR) spectra were recorded using Shimadzu FT. IR-8400S infrared spectro-photometer by $\mathrm{KBr}$ disc, Kufa University. Proton nuclear magnetic resonance $\left({ }^{1} \mathrm{H}-\mathrm{NMR}\right)$ and carbon-13 nuclear magnetic resonance $\left({ }^{13} \mathrm{C}\right.$-NMR) were recorded by Bruker spectrometer, operating at 300 and $400 \mathrm{MHz}$ for ${ }^{1} \mathrm{H}-\mathrm{NMR}$ and $75 \mathrm{MHz}$ for ${ }^{13} \mathrm{C}-\mathrm{NMR}$ with dimethyl sulfoxide-d6 (DMSO-d6), at Abu Ali Center Lab, Mashhad, Iran. Thin-layer chromatography (TLC) was performed on aluminum plates and coated with the layer of $0.25 \mathrm{~mm}$ silica gel; compounds were detected by iodine vapor. Autoclave was used to sterilize agar media, supplied by Prestige Medical- England. Incubator was used to maintain different temperatures required for the growth of organism, supplied by Memert-Germany.

\section{Synthesis of chloro-N-(4-(N-pyrimidin-2- ylsulfamoyl)phenyl) acetamide [A] [14]}

To a stirred mixture of sulfadiazine $(0.01 \mathrm{~mol}, 3 \mathrm{~g})$ and trimethylamine $(1.67 \mathrm{~mL})$ in dimethylformamide (DMF) as solvent. Chloroacetyl chloride $(0.01 \mathrm{~mol}$, $0.95 \mathrm{~mL}$ ) was added drop-wise by micropipette. After the addition was completed, the mixture was stirred for 3 hours without heating. Finally, the solvent was evaporated and the precipitate was filtered, dried and washed with distilled water and ether to generate chloro-N-(4-(N-pyrimidin-2-ylsulfamoyl) phenyl) acetamide $[\mathrm{A}]$. The crystalline precipitates were recrystallized with ethanol. Yield pale yellow $=87 \%$, melting point (m.p.) $=220-222{ }^{\circ} \mathrm{C}$, and retardation factor $R_{\mathrm{f}}=0.75$. The TLC for the reaction was completed by using benzene : methanol at $4: 1$.

\section{Synthesis of 4-(2-aminothiazol-4-ylamino)-N- (pyrimidin-2-yl) benzene sulfonamide [B] [15]}

A mixture of [A] $(0.01 \mathrm{~mol}, 1 \mathrm{~g})$, thiourea (0.01 mol, $0.23 \mathrm{~g}$ ), and anhydrous potassium carbonate $(0.01 \mathrm{~mol}, 0.423 \mathrm{~g})$ in absolute ethanol $(25 \mathrm{~mL})$ was heated under reflux on water bath for 12 hours. The excess of ethanol was removed by distillation and the residue was treated with $5 \%$ sodium carbonate solution to remove acid impurities; the yellow precipitate was filtered, washed with water several times and dried at $50{ }^{\circ} \mathrm{C}$. Finally, the product was crystallized from ethanol. Yield $=78 \%$, m.p. $=178-180^{\circ} \mathrm{C}, R_{\mathrm{f}}=0.58$, and benzene : methanol $=4: 1$. Scheme 1 shows the synthesis of 4-(2-aminothiazol-4-ylamino)-N-

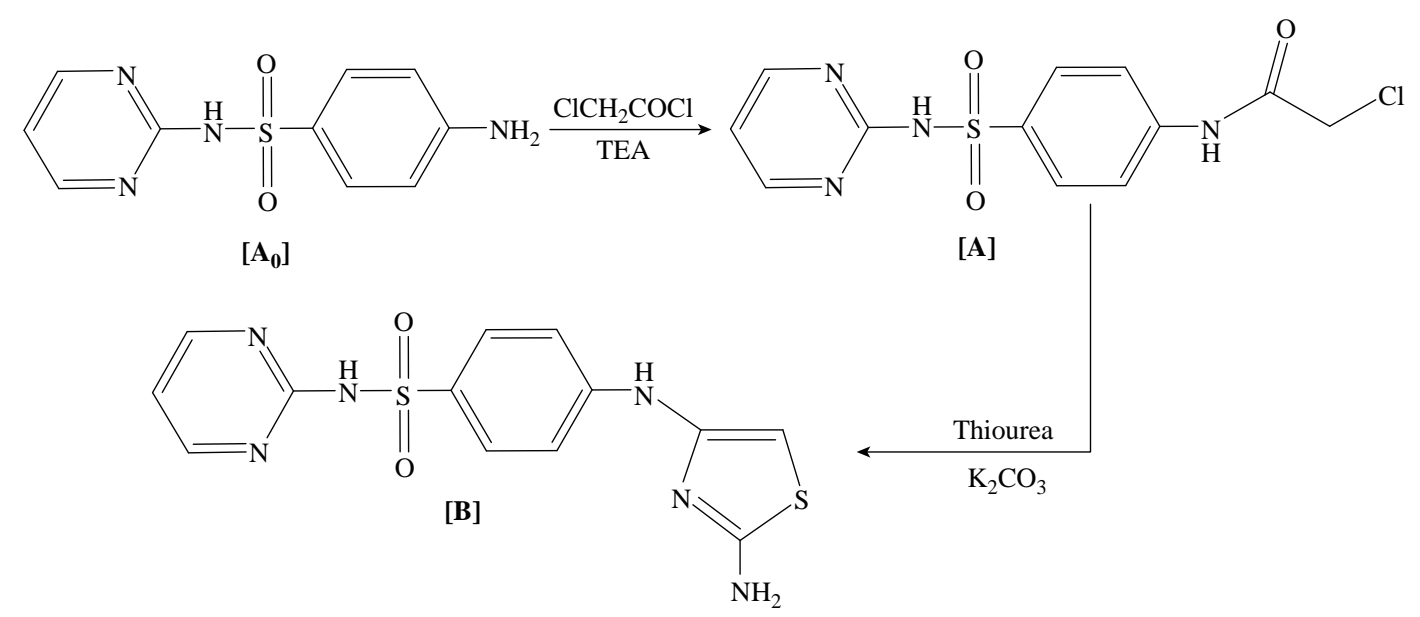

Scheme 1 Chemical reaction of the synthesis of 4-(2-aminothiazol-4-ylamino)-N-(pyrimidin-2-yl) benzene sulfonamide [B]. 
(pyrimidin-2-yl) benzene sulfonamide [B].

\section{General synthesis of Schiff bases $\left[\mathrm{Sh}_{1}-\mathrm{Sh}_{3}\right]$} [16]

A mixture containing 4-(2-aminothiazol-4-ylamino)$\mathrm{N}$-(pyrimidin-2-yl) benzene sulfonamide [B] (0.01 mol) and different aromatic aldehydes $(0.01 \mathrm{~mol})$ in 30 $\mathrm{mL}$ of absolute ethanol with 3 drops of glacial acetic acid was refluxed for 8-10 hours. The reaction mixture was cooled to room temperature. The product was filtered, washed, dried and purified by re-crystallization in hot ethanol to obtain pure compound of Schiff bases [Sh${ }_{1}-\mathrm{Sh}_{3}$ ], (Table 1). Scheme 2 shows the general synthesis of Schiff bases $\left[\mathrm{Sh}_{1}-\mathrm{Sh}_{3}\right]$.

\section{General synthesis of tertiary amide derivatives $\left[\mathrm{D}_{1}-\mathrm{D}_{3}\right][17]$}

In this step, acetyl chloride solution $(0.01 \mathrm{~mol})$ in $10 \mathrm{~mL}$ dry benzene was added dropwise to $0.01 \mathrm{~mol}$ Schiff bases $\left[\mathrm{Sh}_{1}-\mathrm{Sh}_{3}\right.$ ] dissolved in $20 \mathrm{~mL}$ dry benzene. The reaction mixture was stirred for 3 hours, without heating. The product obtained after the evaporation of the solvent was filtered, washed with solution of sodium carbonate of $2 \%$ and re-crystallized from hot ethanol (Table 2). Scheme 3 shows the general synthesis of tertiary amide derivatives [ $\mathrm{D}_{1}$-D3].

\section{Synthesis of 1, 3-bis (hydroxyl methyl) barbituric acids derivatives $\left[E_{1}-E_{2}\right][18]$}

5, 5-diethyl barbituric acid and 5-phenyl, 5-ethyl barbituric acid $(0.01 \mathrm{~mol}, 3 \mathrm{~g})$ was dissolved in ethanol $(25 \mathrm{~mL})$ and distilled water $(10 \mathrm{~mL})$. To this solution, formaldehyde (0.02, 1.19 and $0.95 \mathrm{~mL}$, respectively) was added. The mixture was refluxed at $60-70{ }^{\circ} \mathrm{C}$ for 12 hours. The solvent was evaporated, and the solid precipitate was suspended in water $(10 \mathrm{~mL})$, and extracted with chloroform to give 5,5-diethyl-1,3-bis(hydroxylmethyl) pyrimidine-2,4,6 $(1 \mathrm{H}, 3 \mathrm{H}, 5 \mathrm{H})$-trione $\left[\mathrm{E}_{1}\right]$ and 5-ethyl-5-phenyl-1,3bis(hydroxylmethyl)-pyrimidine-2,4,6(1H,3H,5H) trione $\left[\mathrm{E}_{2}\right]$, Yield white $=85 \%$ and $\left.87 \%\right)$ m.p. $=93-95$, $163-165{ }^{\circ} \mathrm{C}$, and $R_{\mathrm{f}}=0.7$ and 0.65 , respectively.

\section{Synthesis of barbituric acids derivative $\left[F_{1}-F_{3}\right]$ and $\left.\mathbf{G}_{1}-\mathbf{G}_{3}\right][19]$}

Compounds $\left[\mathrm{D}_{1}-\mathrm{D}_{3}\right](0.01 \mathrm{~mol})$ in DMF $(15 \mathrm{~mL})$ was added dropwise to the stirred suspension of 0.01 mol 1,3-bis(hydroxyl methyl) barbituric acids

Table 1 Physical properties of Schiff bases

\begin{tabular}{|c|c|c|c|c|c|}
\hline Compound & General formula & Color & Yield (\%) & M.p. & $R_{\mathrm{f}}$ \\
\hline $\mathrm{Sh}_{1}$ & $\mathrm{C}_{20} \mathrm{H}_{15} \mathrm{BrN}_{6} \mathrm{O}_{2} \mathrm{~S}_{2}$ & Light yellow & 83 & $181-183$ & 0.60 \\
\hline $\mathrm{Sh}_{2}$ & $\mathrm{C}_{22} \mathrm{H}_{21} \mathrm{~N}_{7} \mathrm{O}_{2} \mathrm{~S}_{2}$ & Brown & 79 & 137-139 & 0.73 \\
\hline $\mathrm{Sh}_{3}$ & $\mathrm{C}_{20} \mathrm{H}_{16} \mathrm{~N}_{6} \mathrm{O}_{3} \mathrm{~S}_{2}$ & Dark yellow & 85 & $287-289$ & 0.66 \\
\hline
\end{tabular}

Note: M.p. = Melting point; $R_{\mathrm{f}}=$ Retardation factor

Table 2 Physical properties of tertiary amide derivatives

\begin{tabular}{ccccc}
\hline Compound & General formula & Color & Yield (\%) & M.p. \\
\hline $\mathrm{D}_{1}$ & $\mathrm{C}_{22} \mathrm{H}_{18} \mathrm{BrClN}_{6} \mathrm{O}_{3} \mathrm{~S}_{2}$ & Light yellow & 78 & $175-177$ \\
$\mathrm{D}_{2}$ & $\mathrm{C}_{24} \mathrm{H}_{24} \mathrm{ClN}_{7} \mathrm{O}_{3} \mathrm{~S}_{2}$ & Brown & 71 & 0.72 \\
$\mathrm{D}_{3}$ & $\mathrm{C}_{22} \mathrm{H}_{19} \mathrm{ClN}_{6} \mathrm{O}_{4} \mathrm{~S}_{2}$ & Light yellow & 71 & 0.63 \\
\hline
\end{tabular}

Note: M.p. = Melting point; $R_{\mathrm{f}}=$ Retardation factor

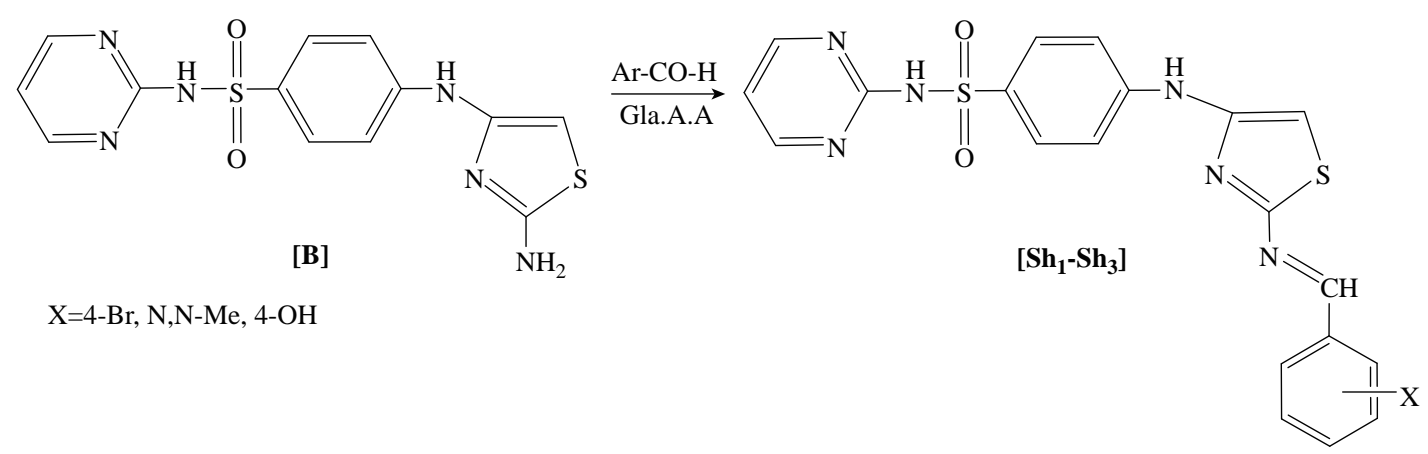

Scheme 2 General synthesis of Schiff bases $\left[\mathrm{Sh}_{1}-\mathrm{Sh}_{3}\right]$. 


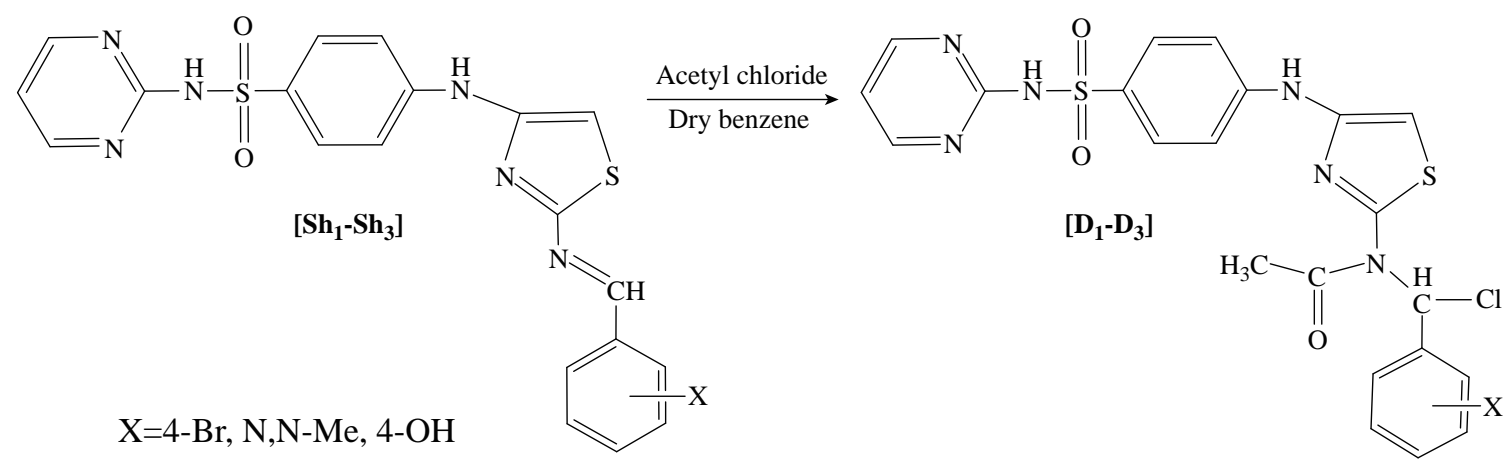

Scheme 3 General synthesis of tertiary amide derivatives $\left[D_{1}-D_{3}\right]$.

derivatives $\left[\mathrm{E}_{1}-\mathrm{E}_{2}\right]$ and $\mathrm{NaOH}$ (0.01 mol) in DMF (15 $\mathrm{mL}$ ). The mixture reaction was refluxed for 5 hours at $60-70{ }^{\circ} \mathrm{C}$. Then, the mixture was diluted with water $(30 \mathrm{~mL})$, and then extracted with ether $(2 \times 15 \mathrm{~mL})$; the combined organic layers were washed successively with water $(20 \mathrm{~mL})$, dried by oven, filtered and evaporated to dryness to give compounds $\left[\mathrm{F}_{1}-\mathrm{F}_{3}\right]$ and $\left[G_{1}-G_{3}\right]$ (Table 3 and 4 ). Scheme 4 shows the synthesis of barbituric acids derivative $\left[F_{1}-F_{3}\right]$ and $\left[G_{1}-G_{3}\right]$.

\section{Test of biological activity}

The synthesized compounds [B], [F $]$, [ $\left.\mathrm{F}_{2}\right],\left[\mathrm{F}_{3}\right],\left[\mathrm{G}_{1}\right]$, $\left[G_{2}\right]$ and $\left[G_{3}\right]$ were tested for their in-vitro antibacterial activity against three types of bacteria, Staphylococcus aureus as a Gram-positive bacterium, Escherichia coli and Pseudomonas aeruginosa as Gram-negative bacteria taking standard gentamicin and penicillin. The antibacterial activity was performed by filter paper disc plate method at concentrations of 0.03 and $0.06 \mu \mathrm{g} /$ $\mathrm{mL}$ using Muller Hinton agar medium, and dimethyl sulfoxide (DMSO) was used as a solvent control. While the antifungal activity for the same compounds was tested against Candida albicans and A. flavus by using filter paper disc plate method and potato dextrose agar (PDA), fluconazole, voriconazole and nystatin were used as standards for the antifungal activity

\section{Results and Discussion}

All the compounds were insoluble in water but soluble in organic solvents as DMF and DMSO. Some synthesized compounds were colored and stable by resonance and having high melting points relatively, which was another evidence on the extent stability. In this study, a series of new barbituric acid derivatives containing thiazole moiety were synthesized from sulfa drug, Sulfadiazine was the starting material for this research, It was converted to the 2-chloro-N(4-(N-pyrimidin-2-ylsulfamoyl) phenyl) acetamide [A] by the reaction with chloroacetyl chloride and trimethylamine in DMF as solvent. The synthesized compound $[\mathrm{A}]$ was characterized by sodium fusion test and the result was positive. The chlorine element was identified using saturated solution of $\mathrm{AgNO}_{3}$ reagent, White precipitate was observed, which was due to the presence of chlorine in compound [A]. FTIR spectrum

Table 3 Physical properties of barbituric acids derivative $\left[\mathrm{F}_{1}-\mathrm{F}_{3}\right]$

\begin{tabular}{cccccc}
\hline Compound & General formula & Color & Yield (\%) & M.p. & $R_{\mathrm{f}}$ \\
\hline $\mathrm{F}_{1}$ & $\mathrm{C}_{32} \mathrm{H}_{33} \mathrm{BrN}_{8} \mathrm{O}_{8} \mathrm{~S}_{2}$ & Light yellow & 79 & $222-224$ & 0.64 \\
$\mathrm{~F}_{2}$ & $\mathrm{C}_{34} \mathrm{H}_{39} \mathrm{~N}_{9} \mathrm{O}_{8} \mathrm{~S}_{2}$ & Dark yellow & 77 & $229-231$ & 0.53 \\
$\mathrm{~F}_{3}$ & $\mathrm{C}_{32} \mathrm{H}_{34} \mathrm{~N}_{8} \mathrm{O}_{9} \mathrm{~S}_{2}$ & White yellowish & 64 & $331-333$ & 0.72 \\
\hline
\end{tabular}

Note: M.p. $=$ Melting point; $R_{\mathrm{f}}=$ Retardation factor

Table 4 Physical properties of barbituric acids derivative $\left[G_{1}-G_{3}\right]$

\begin{tabular}{|c|c|c|c|c|c|}
\hline Comp. Symbol & General formula & Color & Yield (\%) & M.p. & $R_{\mathrm{f}}$ \\
\hline $\mathrm{G}_{1}$ & $\mathrm{C}_{36} \mathrm{H}_{33} \mathrm{BrN}_{8} \mathrm{O}_{8} \mathrm{~S}_{2}$ & Pale yellow & 76 & $238-240$ & 0.62 \\
\hline $\mathrm{G}_{2}$ & $\mathrm{C}_{38} \mathrm{H}_{39} \mathrm{~N}_{9} \mathrm{O}_{8} \mathrm{~S}_{2}$ & Dark yellow & 74 & $130-132$ & 0.74 \\
\hline $\mathrm{G}_{3}$ & $\mathrm{C}_{36} \mathrm{H}_{34} \mathrm{~N}_{8} \mathrm{O}_{9} \mathrm{~S}_{2}$ & Yellow brownish & 75 & $261-263$ & 0.78 \\
\hline
\end{tabular}

Note: M.p. = Melting point; $R_{\mathrm{f}}=$ Retardation factor 


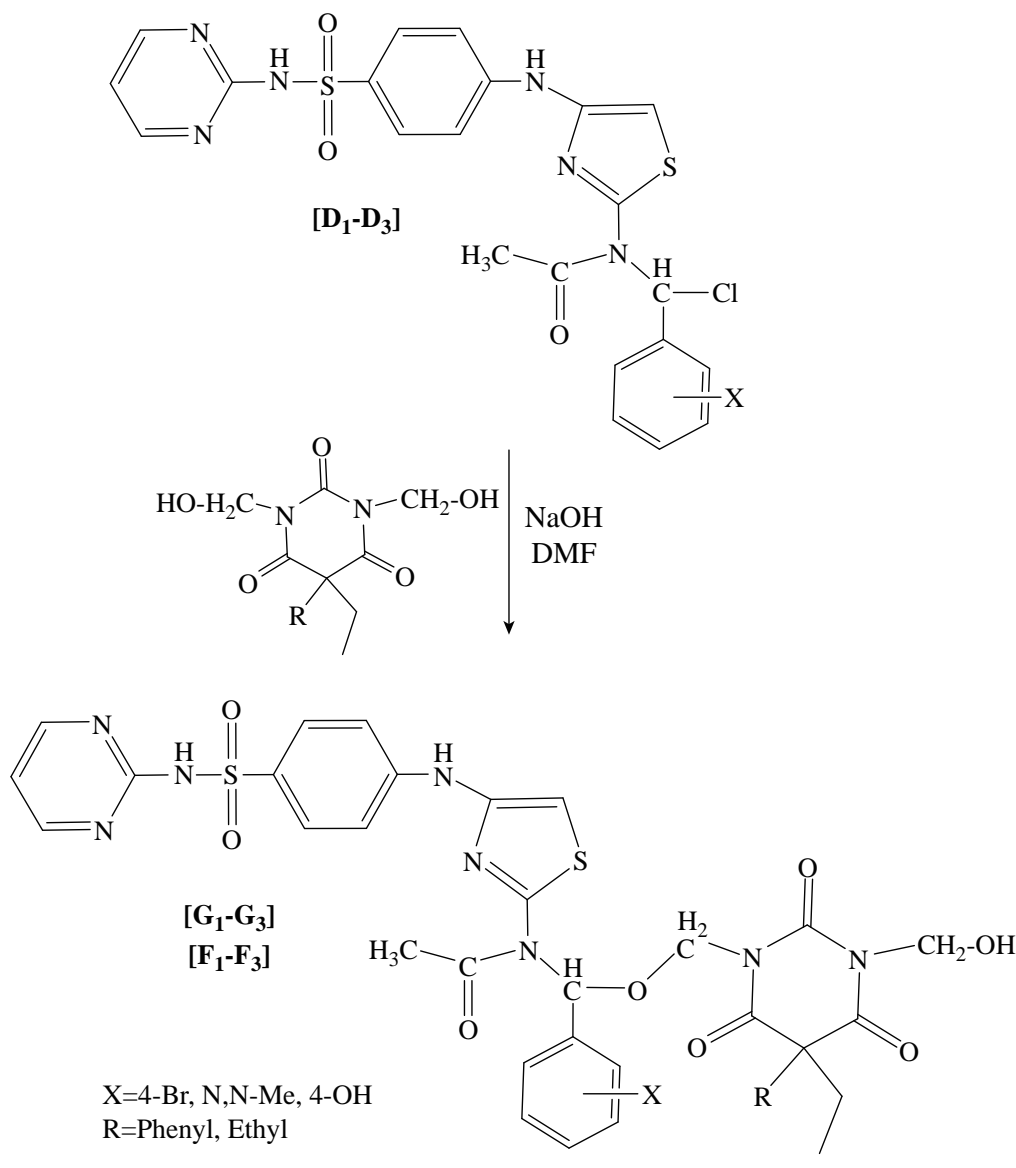

Scheme 4 Synthesis of barbituric acids derivatives $\left[F_{1}-F_{3}\right]$ and $\left[G_{1}-G_{3}\right]$.

in $\mathrm{cm}^{-1}$ : 3381 (-N-H amide), $1670(\mathrm{C}=\mathrm{O}), 1332-1159$ ( $\mathrm{SO}_{2}$ sym.-assy.), 665 (C-Cl) and 1587 (C=C aromatic).

The compound $[\mathrm{B}]$ was prepared by the reaction of [A] with thiourea and anhydrous potassium carbonate in Scheme 1. The structure of [B] was confirmed by FTIR, ${ }^{1} \mathrm{H}-\mathrm{NMR}$ and ${ }^{13} \mathrm{C}-\mathrm{NMR}$.

FTIR spectrum of [B] in $\mathrm{cm}^{-1}$ : 3394-3358 $\left(-\mathrm{NH}_{2}\right)$, $3275(-\mathrm{NH}), 1543$ (C=C thiazole), 1583(C=C aromatic), $1622(\mathrm{C}=\mathrm{N}$ thiazole $)$, and $1662(\mathrm{C}=\mathrm{N}$ pyrimidine).

${ }^{1} \mathrm{H}-\mathrm{NMR}$ spectrum of [B] with DMSO- $d_{6}$ as a solvent: 7.25 (s, $2 \mathrm{H}, \mathrm{NH}_{2}$ ), 5.33 (s, 1H, C-5 thiazole), 7.46-7.71 (m, 4H, Ar-H), 11.48 (s, $1 \mathrm{H}, \mathrm{NH}-\mathrm{SO}_{2}$ ), and 10.46 (s, $1 \mathrm{H}, \mathrm{NH})$.

${ }^{13} \mathrm{C}-\mathrm{NMR}$ spectrum of $[\mathrm{B}]$ with DMSO-d6 ( $\left.\delta \mathrm{ppm}\right)$ : $164.70,160.54,157.71,157.55,150.43,149.84$, 133.41, 128.72, 128.66, 128.15, 128.01, 118.55, 118.35, 116.42, 112.35, 110.91, 110.62, 110.24, 109.78, 40.49, 40.34, 40.28, 40.11, 40.07, 39.86, 39.65, 39.44, and 39.23.

Schiff bases $\left[\mathrm{Sh}_{1}-\mathrm{Sh}_{3}\right]$ were synthesized by the condensation of equimolar quantity of aromatic primary amine [B] with aromatic benzaldehydes: 4-bromobenzaldehyde, 4-dimethylaminobenzaldehyde, and 4-hydroxybenzaldehyde, in the presence of glacial acetic acid as catalyst in absolute ethanol.

FTIR spectrum of 4-(2-(4-bromobenzylideneamino) thiazol-4-ylamino - N-(pyrimidin-2-yl) benzenesulfonamide $\left[\mathrm{Sh}_{1}\right]\left(\mathrm{KBr}, \mathrm{cm}^{-1}\right): 3205(-\mathrm{NH}$ sulfa), 3396 (-NH thiazol), 1674 ( $\mathrm{C}=\mathrm{N}$ imine), 1546 ( $\mathrm{C}=\mathrm{C}$ thiazole), 1583( $\mathrm{C}=\mathrm{C}$ aromatic), $1625(\mathrm{C}=\mathrm{N}$ thiazole), and 516 (C-Br).

${ }^{1} \mathrm{H}-\mathrm{NMR}$ spectrum of $\left[\mathrm{Sh}_{1}\right]$ with DMSO-d6 $(\delta \mathrm{ppm})$ : 8.67 (s, $1 \mathrm{H}, \mathrm{N}=\mathrm{CH}$, 5.75 (s, 1H, C-5 thiazole), 6.787.25 (m, 8H, Ar-H), 11.29 (s, 1H, NH-SO ${ }_{2}$ ), and 10.13 (s, $1 \mathrm{H}, \mathrm{NH}$ ).

${ }^{13} \mathrm{C}$-NMR spectrum of $\left[\mathrm{Sh}_{1}\right]$ with DMSO-d6 $(\delta$ ppm): 178.59, 174.82, 165.91, 161.28, 158.62, 158.10, 157.95, 153.31, 135.47, 132.41, 131.71, 131.35, 131.09, 130.18, 129.78, 129.41, 128.79, 125.80, 120.82, 118.606, 115.61, 112.58, 40.86, 40.58, 40.30, 40.03, 39.75, 39.47, and 39.19.

FTIR spectrum of 4-(2-(4-(dimethylamino) benzylideneamino)thiazol-4-ylamino)-N-(pyrimidin2-yl) benzene sulfonamide $\left[\mathrm{Sh}_{2}\right]$ with $\mathrm{KBr}\left(\mathrm{cm}^{-1}\right): 3442$ (-NH thiazol), 1651 ( $\mathrm{C}=\mathrm{N}$ imine), 1531 ( $\mathrm{C}=\mathrm{C}$ thiazole), 
1577(C=C aromatic), and 1602 ( $\mathrm{C}=\mathrm{N}$ thiazole).

${ }^{1} \mathrm{H}-\mathrm{NMR}$ spectrum of $\left[\mathrm{Sh}_{2}\right]$ with DMSO-d6 ( $\left.\delta \mathrm{ppm}\right)$ : 8.89 (s, $1 \mathrm{H}, \mathrm{N}=\mathrm{CH}_{\text {) }}, 3.32\left(\mathrm{~s}, 6 \mathrm{H},-\mathrm{N}\left(\mathrm{CH}_{3}\right)_{2}\right), 5.33$ (s, 1H, C-5 thiazole), 7.33-7.75 (m, 8H, Ar-H), 11.34 (s, $1 \mathrm{H}, \mathrm{NH}-\mathrm{SO}_{2}$ ), and 10.64 (s, $\left.1 \mathrm{H}, \mathrm{NH}\right)$.

${ }^{13} \mathrm{C}-\mathrm{NMR}$ spectrum of $\left[\mathrm{Sh}_{2}\right]$ with DMSO-d6 $(\delta$ ppm): 172.59, 170.82, 166.91, 163.28, 158.45, 157.84, 157.98, 155.56, 136.36, 132.71, 132.412, 131.52, $131.21,130.43,128.93,128.73,128.46,124.63$, 122.15, 117.68, 115.23, 113.37, 40.45, 40.86, 40.67, 40.29, 40.03, 39.69, 39.34, and 39.12.

FTIR spectrum of 4-(2-(4-hydroxy-3methoxybenzylideneamino)thiazol-4-ylamino)-N(pyrimidin-2-yl) benzene sulfonamide [ $\left.\mathrm{Sh}_{3}\right]$ with $\mathrm{KBr}$ ( $\mathrm{cm}^{-1}$ ): 3421 (-NH thiazol), 1668 (C=N imine), 1548 ( $\mathrm{C}=\mathrm{C}$ thiazole), 1587 ( $\mathrm{C}=\mathrm{C}$ aromatic), $1625(\mathrm{C}=\mathrm{N}$ thiazole), and $3456(\mathrm{OH})$.

${ }^{1} \mathrm{H}-\mathrm{NMR}$ spectrum of $\left[\mathrm{Sh}_{3}\right]$ with DMSO-d6 ( $\left.\delta \mathrm{ppm}\right)$ : 8.95 (s, 1H, N=CH), 12.34 (s,1H,OH), 5.45 (s, 1H, C-5 thiazole), 7.24-7.85 (m, 8H, Ar-H), 11.52 (s, 1H, NH$\mathrm{SO}_{2}$ ), 11.35 (s, $\left.1 \mathrm{H}, \mathrm{NH}\right)$, and 11.78 (s, 1H, OH)..

${ }^{13} \mathrm{C}-\mathrm{NMR}$ spectrum of $\left[\mathrm{Sh}_{3}\right]$ with DMSO-d6 $(\delta$ ppm): 169.95, 169.79, 165.78, 163.65, 158.63, 158.36, $157.86,154.73,135.96,133.78,133.63,131.75$, 131.62, 131.07, 128.63, 129.13, 128.56, 125.67, $122.34,116.79,115.42,113.26,40.97,40.75,40.45$, 40.15, 39.85, 39.57, and 39.36.

Synthesis of compounds $\left[D_{1}-D_{3}\right]$ : Schiff bases reacted with chloroacetyl chloride to yield new tertiary amides in good yield. FTIR of N-((4-bromophenyl) chloromethyl)-N-(4-(4-(N-pyrimidin-2-ylsulfamoyl) phenylamino)thiazol-2-yl) acetamide $\left[\mathrm{D}_{1}\right]$ with $\mathrm{KBr}$ $\left(\mathrm{cm}^{-1}\right)$ : 3387 (-NH), 1672 (C=O amide), 1537 (C=C thiazole), 1589 ( $\mathrm{C}=\mathrm{C}$ aromatic), 1631 ( $\mathrm{C}=\mathrm{N}$ thiazole), and $613(\mathrm{C}-\mathrm{Cl})$.

${ }^{1} \mathrm{H}-\mathrm{NMR}$ spectrum of $\left[\mathrm{D}_{1}\right]$ with DMSO-d6 ( $\left.\delta \mathrm{ppm}\right)$ : 2.09 (s, 3H, O=C-CH ${ }_{3}, 6.57$ (s, 1H, CH-Cl), 5.03 (s, 1H, C-5 thiazole), 6.93-7.94 (m, 8H, Ar-H), 11.77 (s, $1 \mathrm{H}, \mathrm{NH}-\mathrm{SO}_{2}$ ), and 10.45 (s, $\left.1 \mathrm{H}, \mathrm{NH}\right)$.

${ }^{13} \mathrm{C}-\mathrm{NMR}$ spectrum of $\left[\mathrm{D}_{1}\right]$ with DMSO-d6 $(\delta$ ppm): 171.19, 169.01, 166.44, 158.80, 157.41, 157.30, $154.49,143.70,134.25,132.85,131.89,129.64$, 129.29, 119.56, 119.06, 118.67, 118.66, 116.82, 116.61, 77.32, 40.85, 40.57, 40.29, 40.01, 39.74, 39.46, 39.18, and 21.85 .

FTIR of N-(chloro(4-(dimethylamino)phenyl) methyl)-N-(4-(4-(N-pyrimidin-2-ylsulfamoyl) phenylamino)thiazol-2-yl)acetamide $\left[\mathrm{D}_{2}\right]$ with $\mathrm{KBr}$ $\left(\mathrm{cm}^{-1}\right)$ : 3396 (-NH), 1666 (C=O amide), 1548 (C=C thiazole), 1593 ( $\mathrm{C}=\mathrm{C}$ aromatic), 1645 ( $\mathrm{C}=\mathrm{N}$ thiazole), and 613 (C-Cl).

${ }^{1} \mathrm{H}-\mathrm{NMR}$ spectrum of $\left[\mathrm{D}_{2}\right]$ with DMSO-d6 ( $\left.\delta \mathrm{ppm}\right)$ : 2.12 (s, 3H, O=C-CH $\left.{ }_{3}\right), 3.34\left(\mathrm{~s}, 6 \mathrm{H},-\mathrm{N}\left(\mathrm{CH}_{3}\right)_{2}\right), 6.37$ (s,1H,CH-Cl), 5.57 (s, 1H, C-5 thiazole), 7.12-7.84 (m, $8 \mathrm{H}, \mathrm{Ar}-\mathrm{H}), 11.41$ (s, $1 \mathrm{H}, \mathrm{NH}-\mathrm{SO}_{2}$ ), and 11.19 (s, $1 \mathrm{H}$, $\mathrm{NH})$.

${ }^{13} \mathrm{C}-\mathrm{NMR}$ spectrum of $\left[\mathrm{D}_{2}\right]$ with DMSO-d6 $(\delta$ ppm): 165.99, 162.57, 158.70, 157.43, 157.29, 153.41, 143.70, 134.29, 132.85, 131.90, 129.64, 129.29, 119.57, 119.07, 118.67, 118.67, 116.87, 116.62, 115.24, 72.94, 45.93, 40.75, 40.67, 40.10, 40.02, 39.74, 39.465, 39.11, and 23.44.

FTIR of N-(chloro(4-hydroxy-3-methoxyphenyl) methyl)-N-(4-(4-(N-pyrimidin-2-ylsulfamoyl) phenylamino)thiazol-2-yl)acetamide $\left[\mathrm{D}_{3}\right]$ with $\mathrm{KBr}$ $\left(\mathrm{cm}^{-1}\right)$ : 3404 (-NH), 1662 (C=O amide), 1531 (C=C thiazole), 1593 ( $\mathrm{C}=\mathrm{C}$ aromatic), 1627 ( $\mathrm{C}=\mathrm{N}$ thiazole), and 669 (C-Cl).

${ }^{1} \mathrm{H}-\mathrm{NMR}$ spectrum of $\left[\mathrm{D}_{3}\right]$ with DMSO-d6 ( $\left.\delta \mathrm{ppm}\right)$ : 2.23 (s, 3H, O=C-CH ${ }_{3}$ ), 6.39 (s,1H,CH-Cl), 5.63 (s, 1H, C-5 thiazole), 7.09-7.89 (m, 8H, Ar-H), 11.00 (s, $1 \mathrm{H}, \mathrm{NH}-\mathrm{SO}_{2}$ ), 10.99 (s, $\left.1 \mathrm{H}, \mathrm{NH}\right)$, and 12.22 (s, $1 \mathrm{H}$, $\mathrm{OH})$.

${ }^{13} \mathrm{C}-\mathrm{NMR}$ spectrum of $\left[\mathrm{D}_{3}\right]$ with DMSO-d6 $(\delta$ ppm): 174.12, 168.63, 165.69, 158.76, 158.31, 157.63, $157.54,144.50,134.61,133.83,132.52,130.49$, 129.67, 119.74, 119.15, 118.85, 118.42, 116.64, 115.41, 75.47, 40.83, 40.47, 40.19, 40.07, 39.71, 39.49, 39.22, and 24.62 .

1,3-bis(hydroxyl methyl)barbituric acids derivatives $\left[E_{1}-E_{2}\right]$ were prepared from reaction of 5,5-diethyl barbituric acid and 5-phenyl,5-ethyl barbituric acid with formaldehyde.

FTIR spectrum for $\left[\mathrm{E}_{1}\right]$ with $\mathrm{KBr}\left(\mathrm{cm}^{-1}\right)$ : $3452(-\mathrm{OH})$, 1674 (-C=O amide), (1714, 1766) (-C=O barbituric acid), and for compound $\left[\mathrm{E}_{2}\right]$ with $\mathrm{KBr}\left(\mathrm{cm}^{-1}\right): 3464$ (-OH), 1635 (-C=O amide), and 1724 (-C=O barbituric acid).

Williamson etherification of $\left[\mathrm{D}_{1}-\mathrm{D}_{3}\right]$ with $\left[\mathrm{E}_{1}-\mathrm{E}_{2}\right]$ in the presence of $\mathrm{NaOH}$ and $\mathrm{DMF}$ as solvent afforded $\left[F_{1}-F_{3}\right]$ and $\left[G_{1}-G_{3}\right]$, in very good yields.

FTIR of $\left[\mathrm{F}_{1}\right]$ with $\mathrm{KBr}\left(\mathrm{cm}^{-1}\right)$ : $3412(-\mathrm{OH})$, overlap (-NH), 1024 (-C-O), 1668 (-C=O amide), (1780, 1691) (-C=O barbituric acid), 1627 ( $\mathrm{C}=\mathrm{N}$ thiazole), 1593 
(C=C aromatic), and 619 (C-Br).

${ }^{1} \mathrm{H}-\mathrm{NMR}$ spectrum of $\left[\mathrm{F}_{1}\right]$ with DMSO-d6 ( $\left.\delta \mathrm{ppm}\right)$ : 1.72 (q, 4H, $\mathrm{CH}_{2}$ barbituric acid), 0.68 (t, 6H, $\mathrm{CH}_{3}$ barbituric acid), 4.29 (s, 2H, O- $\mathrm{CH}_{2}-\mathrm{N}$ ), 4.16 (s, 2H, $\mathrm{N}-\mathrm{CH}_{2}$ ), 6.41 (s, 1H, N-CH), 2.03 (s, 3H, CO-CH ${ }_{3}$, 7.17-7.97 (m, 8H, Ar-H), 5.41 (s, 1H, C-5 thiazole), 10.31 (s, 1H, NH), and 10.70 (s, 1H, NH pyrimidine).

${ }^{13} \mathrm{C}-\mathrm{NMR}$ spectrum of $\left[\mathrm{F}_{1}\right]$ with DMSO-d6 $(\delta$ ppm): 178.85, 169.02, 164.91, 158.06, 157.72, 141.41, $140.71,128.68,128.11,127.92,118.29,118.04$, 110.00, 55.50, 40.81, 40.53, 40.25, 39.98, 39.70, 39.42, 39.14, 31.89, 24.48, and 9.97.

FTIR of $\left[\mathrm{F}_{2}\right]$ with $\mathrm{KBr}\left(\mathrm{cm}^{-1}\right)$ : $3402(-\mathrm{OH})$, overlap (-NH), 1043 (-C-O), 1668 (-C=O amide), 1695 (-C=O barbituric acid), 3184 ( $\mathrm{HC}=\mathrm{N}$ thiazole), 1583 ( $\mathrm{C}=\mathrm{C}$ aromatic), and 1546 ( $\mathrm{C}=\mathrm{C}$ thiazole).

${ }^{1} \mathrm{H}-\mathrm{NMR}$ spectrum of $\left[\mathrm{F}_{2}\right]$ with DMSO-d6 ( $\left.\delta \mathrm{ppm}\right)$ : 1.69 (q, 4H, $\mathrm{CH}_{2}$ barbituric acid), 0.66 (t, 6H, $\mathrm{CH}_{3}$ barbituric acid), 4.28 (s, 2H, O- $\left.\mathrm{CH}_{2}-\mathrm{N}\right), 4.24$ (s, 2H, $\mathrm{N}-\mathrm{CH}_{2}$ ), 6.38 (s, 1H, N-CH), 2.04 (s, 3H, CO-CH ${ }_{3}$, 3.05 (s, 6H, N( $\left.\left.\mathrm{CH}_{3}\right)_{2}\right)$, 6.78-7.72 (m, 8H, Ar-H), 5.22 (s, 1H, C-5 thiazole), 10.43 (s, 1H, NH), and 11.35 (s, $1 \mathrm{H}$, NH pyrimidine).

${ }^{13} \mathrm{C}-\mathrm{NMR}$ spectrum of $\left[\mathrm{F}_{2}\right]$ with DMSO-d6 $(\delta$ ppm): 181.24, 171.27, 164.93, 164.91, 158.07, 147.65, $140.71,132.11,128.11,127.93,118.30,118.04$, 110.27, 56.42, 40.82, 40.53, 40.25, 39.98, 39.70, 39.42, 39.14, 30.91, 22.91, and 8.65.

FTIR of $\left[\mathrm{F}_{3}\right]$ with $\mathrm{KBr}\left(\mathrm{cm}^{-1}\right)$ : $3408(-\mathrm{OH})$, overlap (-NH), 1043 (-C-O), 1666 (-C=O amide), 1697 (-C=O barbituric acid), 3184 (HC=N thiazole), and 1570 (C=C aromatic).

${ }^{1} \mathrm{H}-\mathrm{NMR}$ spectrum of $\left[\mathrm{F}_{3}\right]$ with DMSO-d6 $(\delta \mathrm{ppm})$ : 1.74 (q, 4H, $\mathrm{CH}_{2}$ barbituric acid), 0.69 (t, 6H, $\mathrm{CH}_{3}$ barbituric acid), 4.31 (s, 2H, O- $\mathrm{CH}_{2}-\mathrm{N}$ ), 4.28 (s, 2H, $\mathrm{N}-\mathrm{CH}_{2}$ ), 6.52 (s, 1H, N-CH), 2.06 (s, 3H, CO-CH ${ }_{3}$ ), 7.15-7.84 (m, 8H, Ar-H), 5.38 (s, 1H, C-5 thiazole), 10.41 (s, 1H, NH), 11.28 (s, 1H, NH pyrimidine), and 11.86 (s, 1H, OH).

${ }^{13} \mathrm{C}-\mathrm{NMR}$ spectrum of $\left[\mathrm{F}_{3}\right]$ with DMSO-d6 $(\delta$ ppm): 175.65, 167.57, 163.87, 158.06, 157.72, 141.41, $140.71,128.68,128.11,127.92,118.29,118.04$, 110.22, 55.51, 40.81, 40.53, 40.25, 39.98, 39.70, 39.42, 39.14, 31.74, 22.91, and 7.89.

FTIR of $\left[\mathrm{G}_{1}\right]$ with $\mathrm{KBr}\left(\mathrm{cm}^{-1}\right)$ : $3433(-\mathrm{OH})$, overlap (-NH), 1049 (-C-O), 1666 (-C=O amide), 1693 (-C=O barbituric acid), 1631 ( $\mathrm{C}=\mathrm{N}$ thiazole), 1587 ( $\mathrm{C}=\mathrm{C}$ aromatic), and 619 (C-Br).

${ }^{1} \mathrm{H}-\mathrm{NMR}$ spectrum of $\left[\mathrm{G}_{1}\right]$ with DMSO-d6 ( $\left.\delta \mathrm{ppm}\right)$ : 2.17 (q, 4H, $\mathrm{CH}_{2}$ barbituric acid), 0.78 (t, 3H, $\mathrm{CH}_{3}$ barbituric acid), 4.52 (s, 2H, O- $\mathrm{CH}_{2}-\mathrm{N}$ ), 4.28 (s, 2H, $\mathrm{N}-\mathrm{CH}_{2}$ ), 6.73 (s, 1H, N-CH), 2.01 (s, 3H, CO-CH ${ }_{3}$ ), 7.18-7.95 (m, 12H, Ar-H), 5.23 (s, 1H, C-5 thiazole), 10.50 (s, 1H, NH), and 11.26 (s, 1H, NH pyrimidine).

${ }^{13} \mathrm{C}-\mathrm{NMR}$ spectrum of $\left[\mathrm{G}_{1}\right]$ with DMSO-d6 $(\delta$ ppm): 173.45, 169.72, 164.60, 158.62, 158.23, 141.52, 141.15, 128.73, 128.43, 127.74, 117.96, 117.67, 111.15, 53.48, 40.84, 40.52, 40.28, 39.83, 39.62, 39.37, 39.19, 29.94, 23.78, and 8.63.

FTIR of $\left[\mathrm{G}_{2}\right]$ with $\mathrm{KBr}\left(\mathrm{cm}^{-1}\right): 3390(-\mathrm{OH})$, overlap (-NH), 1047 (-C-O), 1664 (-C=O amide), 1643 (C=N thiazole), and 1591 ( $\mathrm{C}=\mathrm{C}$ aromatic).

${ }^{1} \mathrm{H}-\mathrm{NMR}$ spectrum of $\left[\mathrm{G}_{2}\right]$ with DMSO-d6 ( $\left.\delta \mathrm{ppm}\right)$ : 2.16 (q, 4H, $\mathrm{CH}_{2}$ barbituric acid), 0.76 (t, 3H, $\mathrm{CH}_{3}$ barbituric acid), 4.64 (s, 2H, O- $\mathrm{CH}_{2}-\mathrm{N}$ ), 4.29 (s, 2H, $\mathrm{N}-\mathrm{CH}_{2}$ ), 6.37 (s, 1H, N-CH), 2.03 (s, 3H, CO-CH ${ }_{3}$, 3.05 (s, 6H, N( $\left.\left.\mathrm{CH}_{3}\right)_{2}\right), 7.12-7.77$ (m, 12H, Ar-H), 5.31 (s, 1H, C-5 thiazole), 10.39 (s, 1H, NH), and 11.66 (s, $1 \mathrm{H}, \mathrm{NH}$ pyrimidine).

${ }^{13} \mathrm{C}-\mathrm{NMR}$ spectrum of $\left[\mathrm{G}_{2}\right]$ with DMSO-d6 $(\delta$ ppm): 177.03, 168.51, 165.47, 159.21, 158.73, 140.62, $141.23,128.59,128.38,128.13,118.85,117.45$, 111.34, 51.47, 40.81, 40.53, 40.26, 39.85, 39.68, 39.32, 39.11, 28.87, 24.37, and 8.72.

FTIR of $\left[\mathrm{G}_{3}\right]$ with $\mathrm{KBr}\left(\mathrm{cm}^{-1}\right)$ : $3408(-\mathrm{OH})$, overlap (-NH), 1039 (-C-O), 1664 (-C=O amide), 1707 overlap (-C=O barbituric acid), 1643 ( $\mathrm{C}=\mathrm{N}$ thiazole), and 1585 ( $\mathrm{C}=\mathrm{C}$ aromatic).

${ }^{1} \mathrm{H}-\mathrm{NMR}$ spectrum of $\left[\mathrm{G}_{3}\right]$ with DMSO-d6 ( $\left.\delta \mathrm{ppm}\right)$ : 2.19 (q, 4H, $\mathrm{CH}_{2}$ barbituric acid), 0.79 (t, 3H, $\mathrm{CH}_{3}$ barbituric acid), 4.52 (s, 2H, O- $\mathrm{CH}_{2}-\mathrm{N}$ ), 4.28 (s, 2H, $\mathrm{N}-\mathrm{CH}_{2}$ ), 6.40 (s, 1H, N-CH), 2.06 (s, 3H, CO-CH ${ }_{3}$, 7.19-7.97 (m, 12H, Ar-H), 5.34 (s, 1H, C-5 thiazole), 10.38 (s, 1H, NH), 11.26 (s, 1H, NH pyrimidine), and 11.66 (s, 1H, OH).

${ }^{13} \mathrm{C}-\mathrm{NMR}$ spectrum of $\left[\mathrm{G}_{3}\right]$ with DMSO-d6 $(\delta$ ppm): 169.45, 167.93, 165.62, 158.91, 158.63, 141.79, $141.54,129.69,128.82,128.36,117.72,117.41$, 110.94, 55.74, 40.84, 40.73, 40.58, 39.83, 39.64, 39.53, 39.16, 26.68, 22.79, and 8.57.

\section{Antimicrobial activity}

Antibacterial activity of synthesized compounds was tested against three types of bacteria: Staphylococcus aureus as a Gram-positive bacterium, Escherichia 
coli and Pseudomonas aeruginosa as Gram-negative bacteria by using Muller Hinton agar medium. The prepared compounds $[\mathrm{B}],\left[\mathrm{F}_{1}\right],\left[\mathrm{F}_{2}\right],\left[\mathrm{G}_{1}\right]$ and $\left[\mathrm{G}_{2}\right]$ were measured for zone of inhibition around each disc. Solvent control (DMSO) did not inhibit bacteria or fungi. The test results are presented in Table 5 and Fig. 19.

Table 5 shows that $[B]$ and $\left[G_{1}\right]$ were highly active against $S$. aureous, $P$. aeruginosa and E.coli at 0.03 and $0.06 \mathrm{mg} / \mathrm{mL}$. Compound $\left[\mathrm{F}_{2}\right]$ was highly active against $P$. aeruginosa and E. coli at $0.06 \mathrm{mg} / \mathrm{mL}$, and it was moderately active against them at $0.03 \mathrm{mg} /$ $\mathrm{mL}$, while it was inactive against $S$. aureous for both concentrations.

Compound $\left[G_{2}\right]$ was highly active against $S$. aureous and E. coli at at $0.06 \mathrm{mg} / \mathrm{mL}$, and it was moderately active against them at $0.03 \mathrm{mg} / \mathrm{mL}$, while it was inactive against $P$. aeruginosa.

Antifungal activity of the synthesized compounds was tested against two types of fungi, Candida albicans and Aspergillus flavus by using potato dextrose agar (PDA) medium. The test results are presented in Table 6 and Fig. 2. Compounds [B], [G $\left.\mathrm{G}_{1}\right]$ and $\left[G_{2}\right]$ were highly active against Candida albicans at 0.03 and $0.06 \mathrm{mg} / \mathrm{mL}$, while compound $\left[\mathrm{F}_{1}\right]$ and $\left[\mathrm{F}_{2}\right]$ were inactive against the same fungus.

In Aspergillus flavus, compounds [B] and $\left[\mathrm{G}_{2}\right]$ were inactive at 0.03 and $0.06 \mathrm{mg} / \mathrm{mL}$, while $\left[\mathrm{F}_{1}\right]$ and $\left[\mathrm{G}_{1}\right]$ were moderately active at 0.03 and $0.06 \mathrm{mg} / \mathrm{mL}$. Compound $\left[\mathrm{F}_{2}\right]$ was moderately active at $0.03 \mathrm{mg} / \mathrm{mL}$ and highly active at $0.06 \mathrm{mg} / \mathrm{mL}$.

FTIR spectra of compounds $[\mathrm{A}],[\mathrm{B}],\left[\mathrm{Sh}_{1}\right],\left[\mathrm{D}_{1}\right]$, $\left[\mathrm{E}_{1}\right]$, $\left[\mathrm{F}_{1}\right]$ and $\left[\mathrm{G}_{1}\right]$ was shown in Fig. 3, 4, 5, 6 7, 8 and 9, respectively.

${ }^{1} \mathrm{H}-\mathrm{NMR}$ spectra of compounds [B], [Sh $\left.\mathrm{Sh}_{1}\right],\left[\mathrm{D}_{1}\right]$, $\left[F_{1}\right]$ and $\left[G_{1}\right]$ are shown in Fig. $10,11,12,13,14$, respectively. ${ }^{13} \mathrm{C}-\mathrm{NMR}$ spectra of compounds [B], $\left[\mathrm{Sh}_{1}\right],\left[\mathrm{D}_{1}\right]$ and $\left[\mathrm{F}_{1}\right]$ are shown in Fig. $15,16,17,18$,

Table 5 Antibacterial activity of some synthesized compounds

\begin{tabular}{|c|c|c|c|c|c|c|c|}
\hline & \multirow{3}{*}{ Sample } & \multicolumn{6}{|c|}{ Inhibition zone diameter (mm/mg sample) } \\
\hline & & \multicolumn{2}{|c|}{ S. aureus } & \multicolumn{2}{|c|}{ P. aeruginosa } & \multicolumn{2}{|c|}{ E. coli } \\
\hline & & 0.03 & 0.06 & 0.03 & 0.06 & 0.03 & 0.06 \\
\hline & B & 20 & 28 & 20 & 25 & 20 & 20 \\
\hline & $\mathrm{F}_{1}$ & $\mathrm{R}$ & $\mathrm{R}$ & 20 & 28 & 20 & 28 \\
\hline & $\mathrm{F}_{2}$ & $\mathrm{R}$ & $\mathrm{R}$ & 15 & 20 & 15 & 20 \\
\hline & $\mathrm{G}_{1}$ & 28 & 30 & 15 & 20 & 17 & 20 \\
\hline & $\mathrm{G}_{2}$ & 15 & 20 & $\mathrm{R}$ & $\mathrm{R}$ & 15 & 20 \\
\hline \multirow{3}{*}{ Standard } & Gentamicin & \multicolumn{2}{|c|}{31} & \multicolumn{2}{|c|}{28} & \multicolumn{2}{|c|}{22} \\
\hline & Penicillin & \multicolumn{2}{|c|}{32} & \multicolumn{2}{|c|}{20} & \multicolumn{2}{|c|}{23} \\
\hline & Control: DMSO & \multicolumn{2}{|c|}{$\mathrm{R}$} & \multicolumn{2}{|c|}{$\mathrm{R}$} & \multicolumn{2}{|c|}{$\mathrm{R}$} \\
\hline
\end{tabular}

Table 6 Antifungal activity of some synthesized compounds

\begin{tabular}{|c|c|c|c|c|c|}
\hline & \multirow{3}{*}{ Sample } & \multicolumn{4}{|c|}{ Inhibition zone diameter (mm/mg sample) } \\
\hline & & \multicolumn{2}{|c|}{ Candida alicans } & \multicolumn{2}{|c|}{ A. flavus } \\
\hline & & 0.03 & 0.06 & 0.03 & 0.06 \\
\hline B & & 22 & 25 & $\mathrm{R}$ & $\mathrm{R}$ \\
\hline $\mathrm{F}_{1}$ & & $\mathrm{R}$ & $\mathrm{R}$ & 10 & 15 \\
\hline $\mathrm{F}_{2}$ & & $\mathrm{R}$ & $\mathrm{R}$ & 15 & 22 \\
\hline $\mathrm{G}_{1}$ & & 20 & 23 & 15 & 15 \\
\hline $\mathrm{G}_{2}$ & & 22 & 28 & $\mathrm{R}$ & $\mathrm{R}$ \\
\hline \multirow{4}{*}{ Standard } & Fluconazole & \multicolumn{2}{|c|}{22} & \multicolumn{2}{|c|}{16} \\
\hline & Voriconazole & \multicolumn{2}{|c|}{28} & \multicolumn{2}{|c|}{--} \\
\hline & Nystatin & \multicolumn{2}{|c|}{--} & \multicolumn{2}{|c|}{13} \\
\hline & Control: DMSO & \multicolumn{2}{|c|}{$\mathrm{R}$} & \multicolumn{2}{|c|}{$\mathrm{R}$} \\
\hline
\end{tabular}



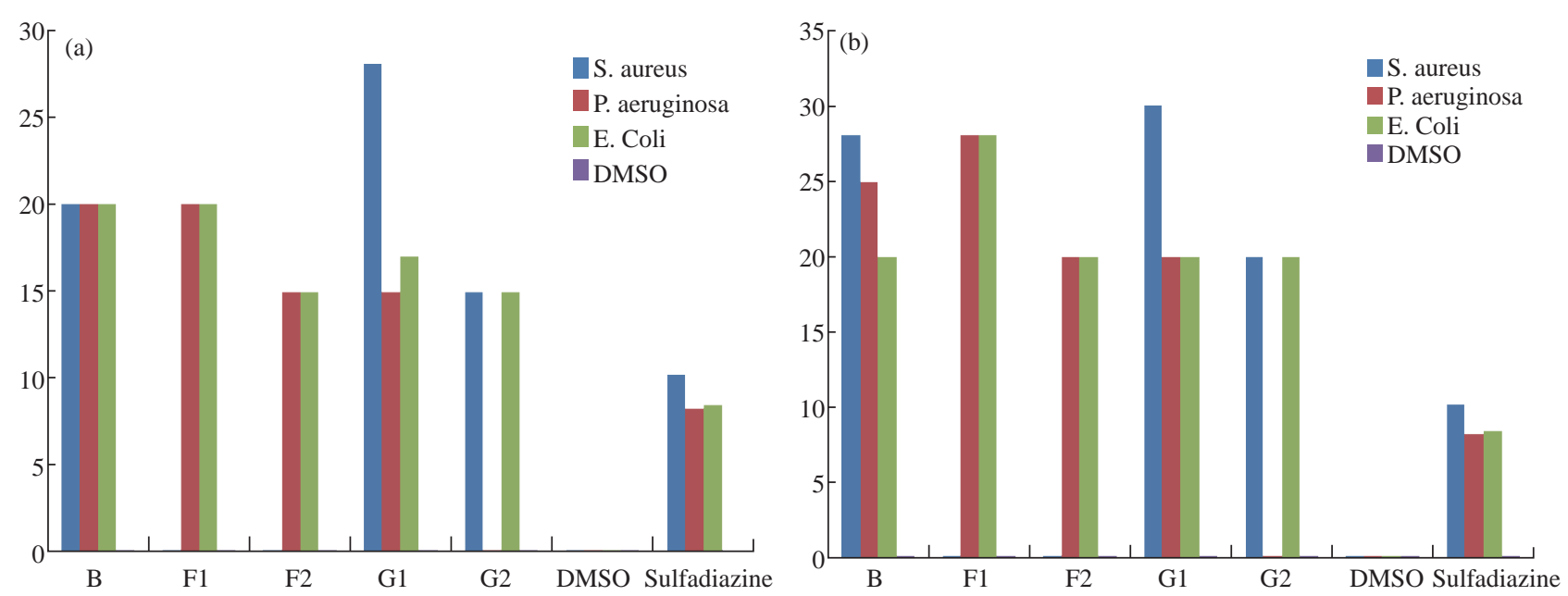

Fig. 1 Antibacterial activity of some synthesized compounds at (a) $0.03 \mathrm{mg} / \mathrm{mL}$ and (b) $0.06 \mathrm{mg} / \mathrm{mL}$.
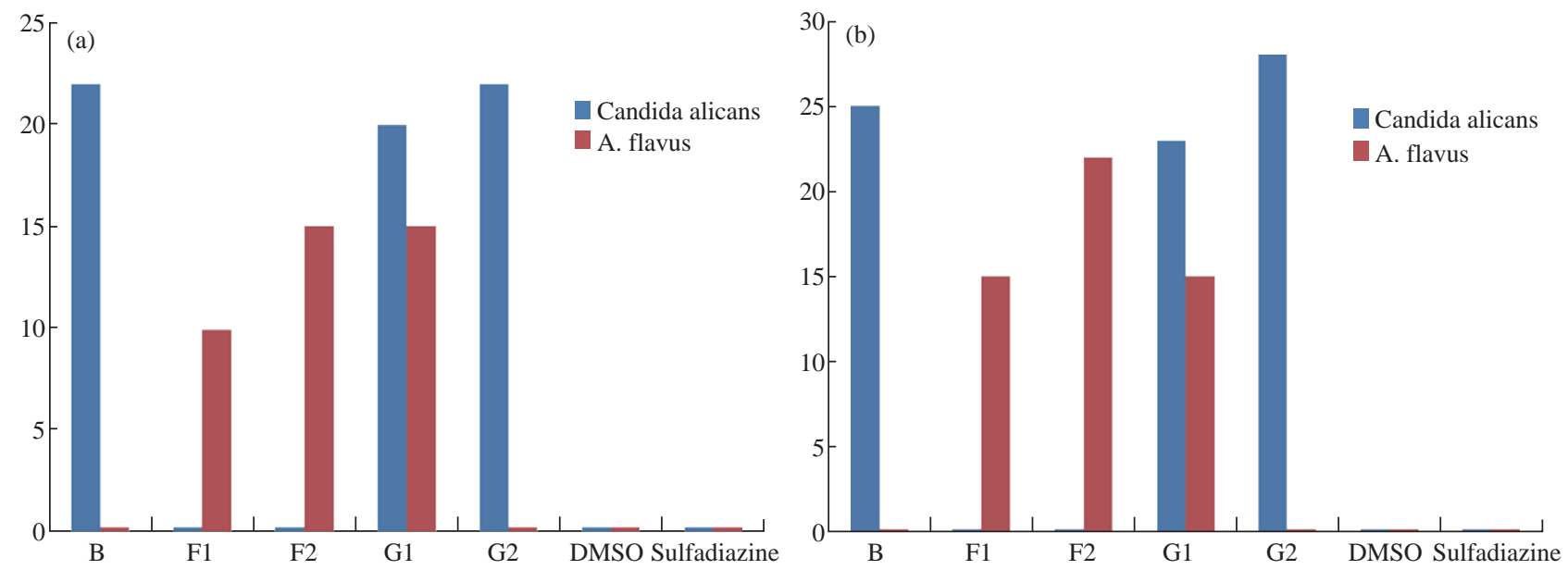

Fig. 2 Antifungal activity of some synthesized compounds at (a) $0.03 \mathrm{mg} / \mathrm{mL}$ and (b) $0.06 \mathrm{mg} / \mathrm{mL}$.

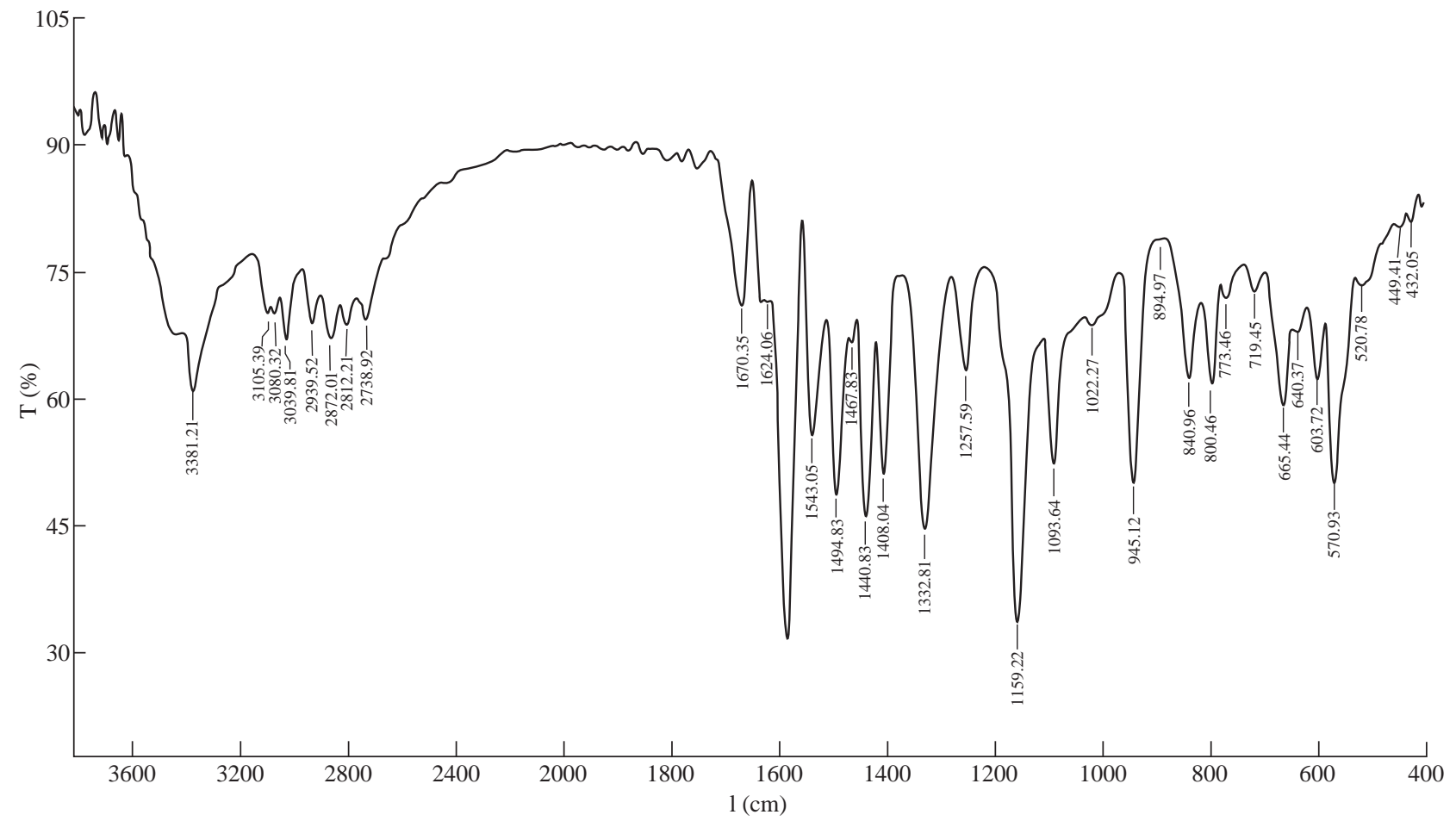

Fig. 3 FTIR spectrum of compound [A]. 


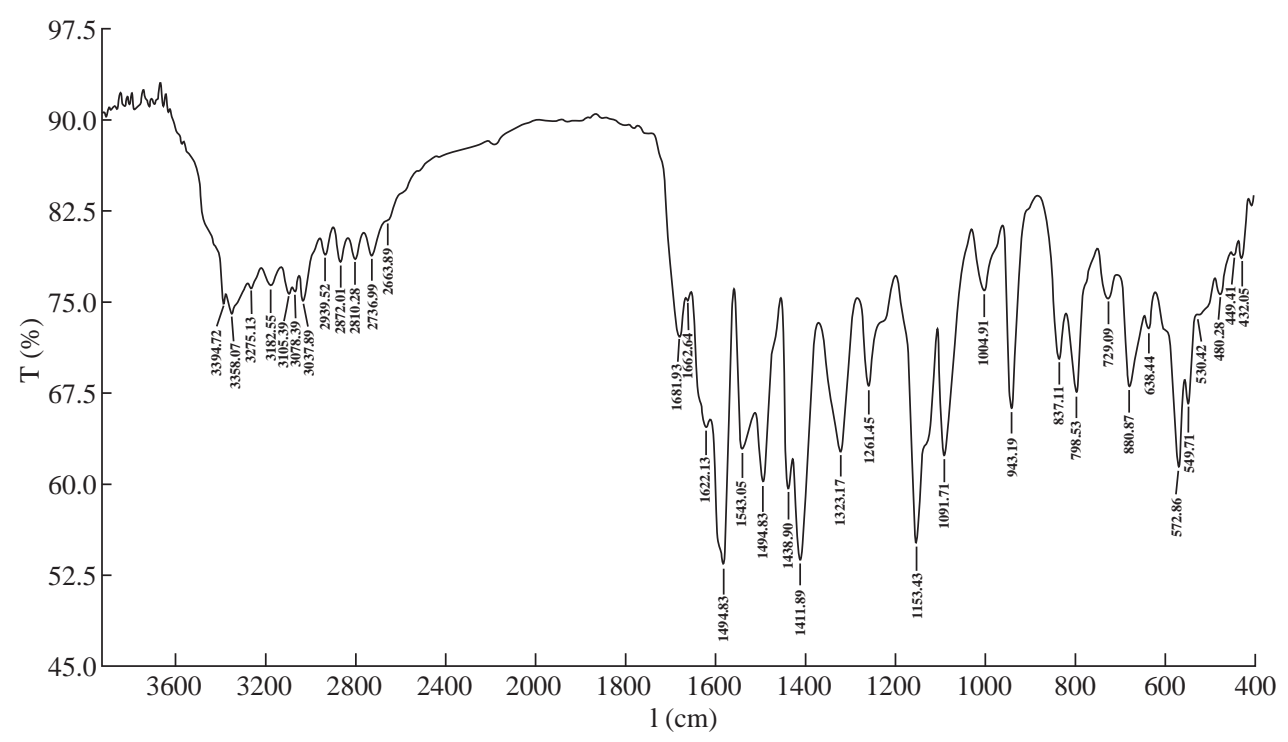

Fig. 4 FTIR spectrum of compound [B].

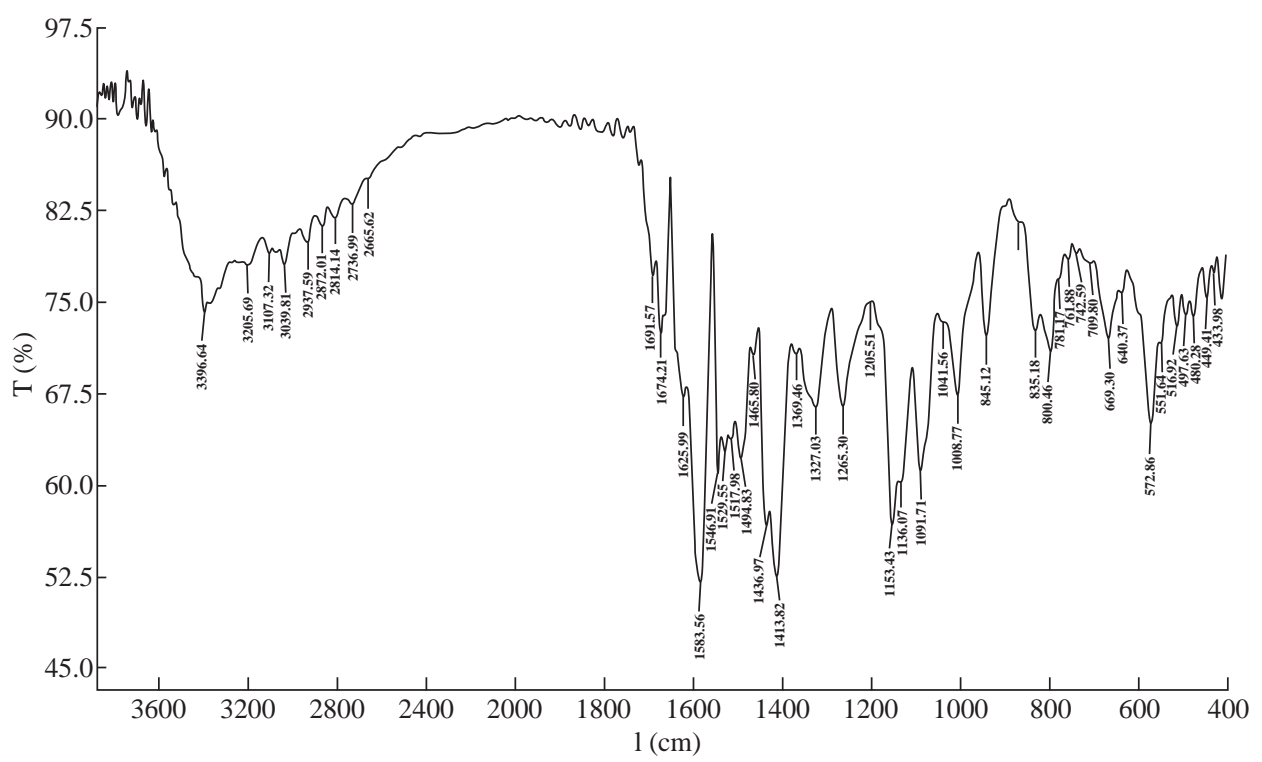

Fig. 5 FTIR spectrum of compound $\left[\mathrm{Sh}_{1}\right]$.

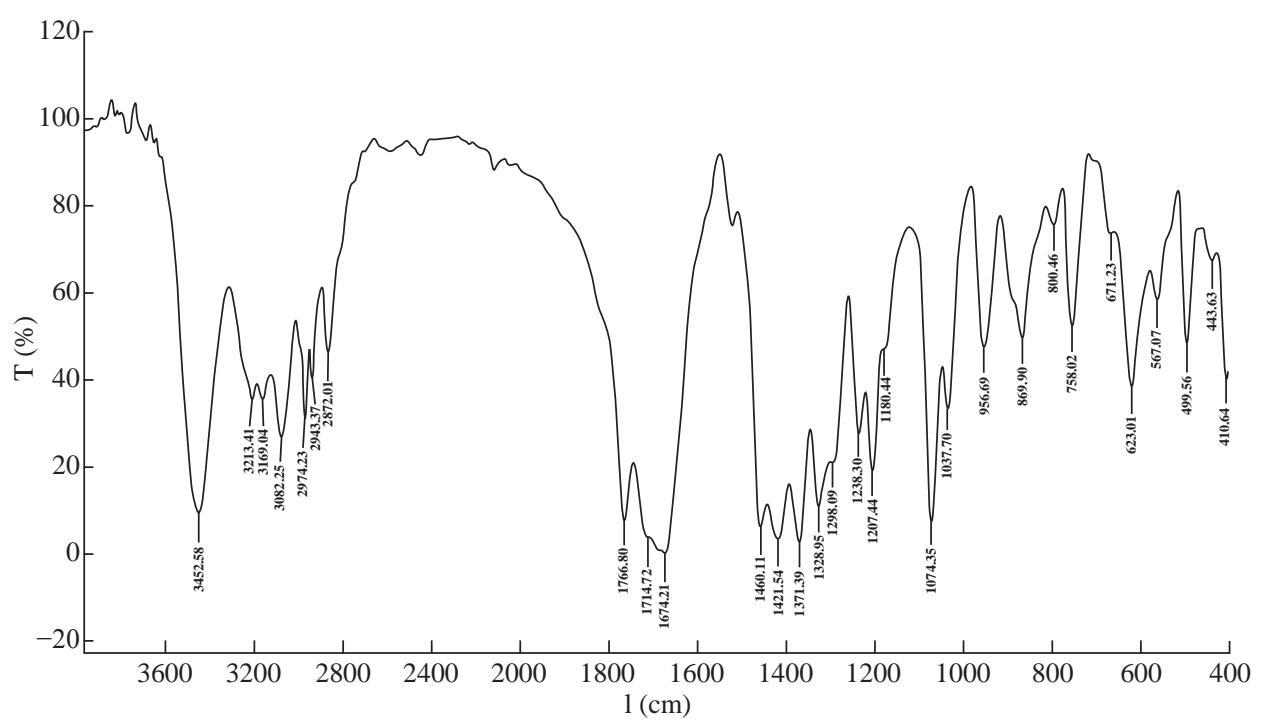

Fig. 6 FTIR spectrum of compound $\left[D_{1}\right]$. 


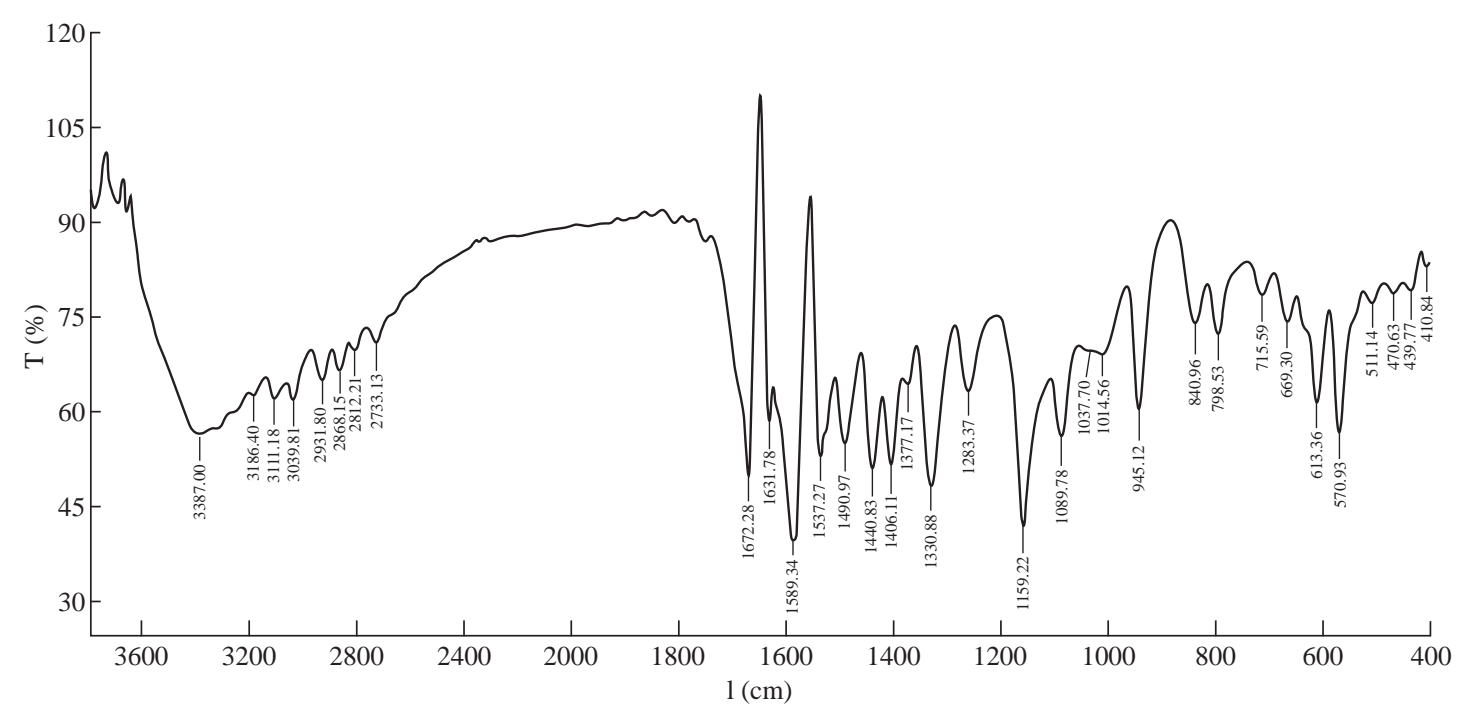

Fig. 7 FTIR spectrum of compound $\left[\mathrm{E}_{1}\right]$.

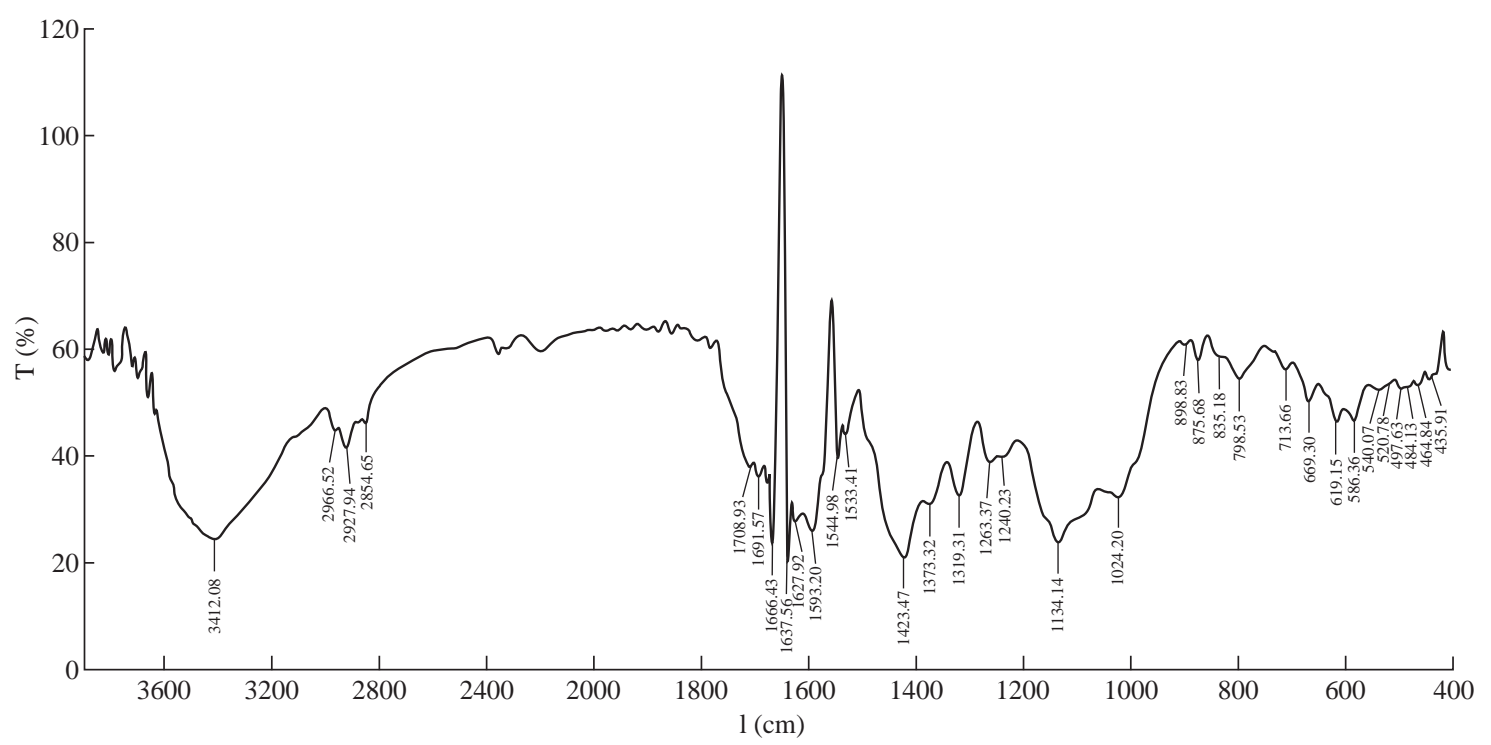

Fig. 8 FTIR spectrum of compound $\left[\mathrm{F}_{1}\right]$.

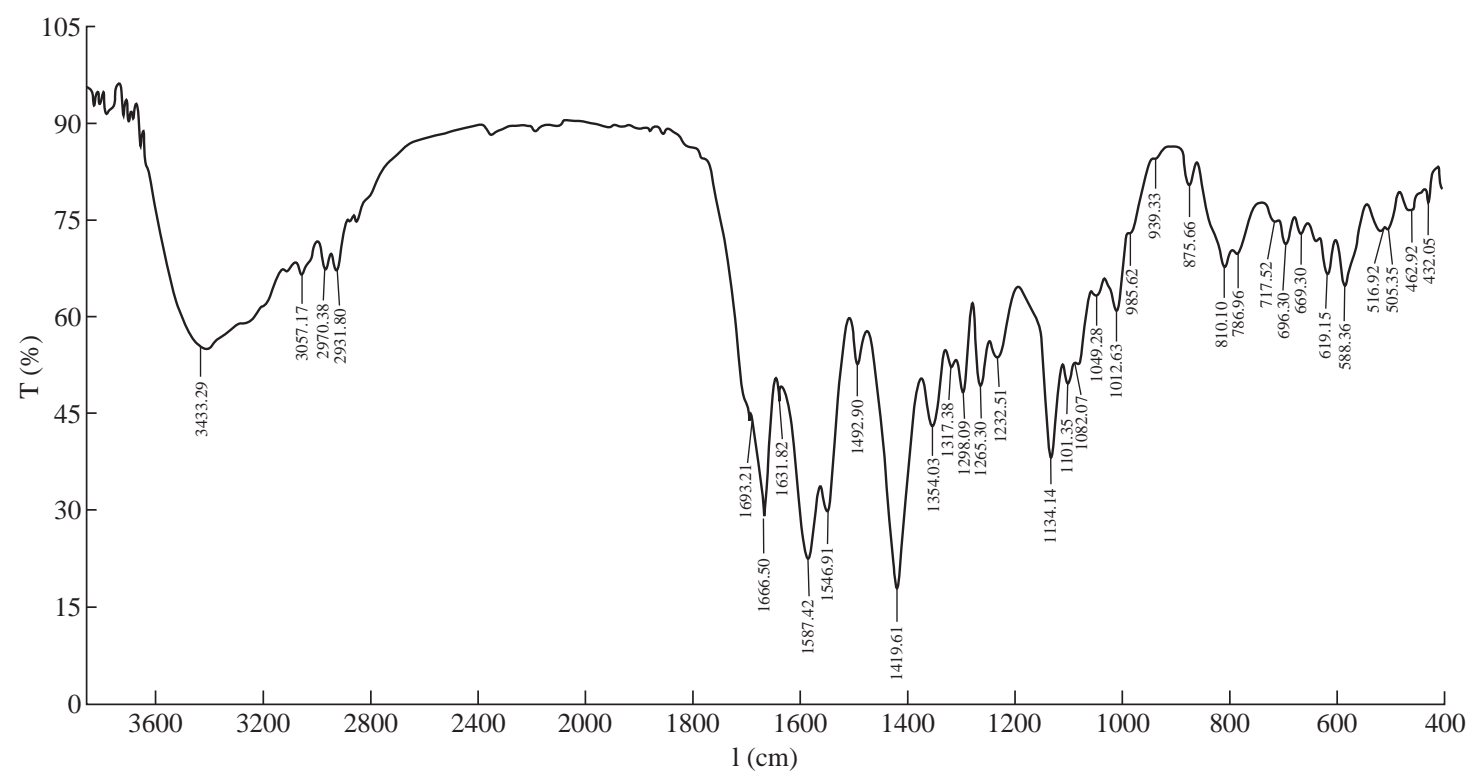

Fig. 9 FTIR spectrum of compound [F]. 


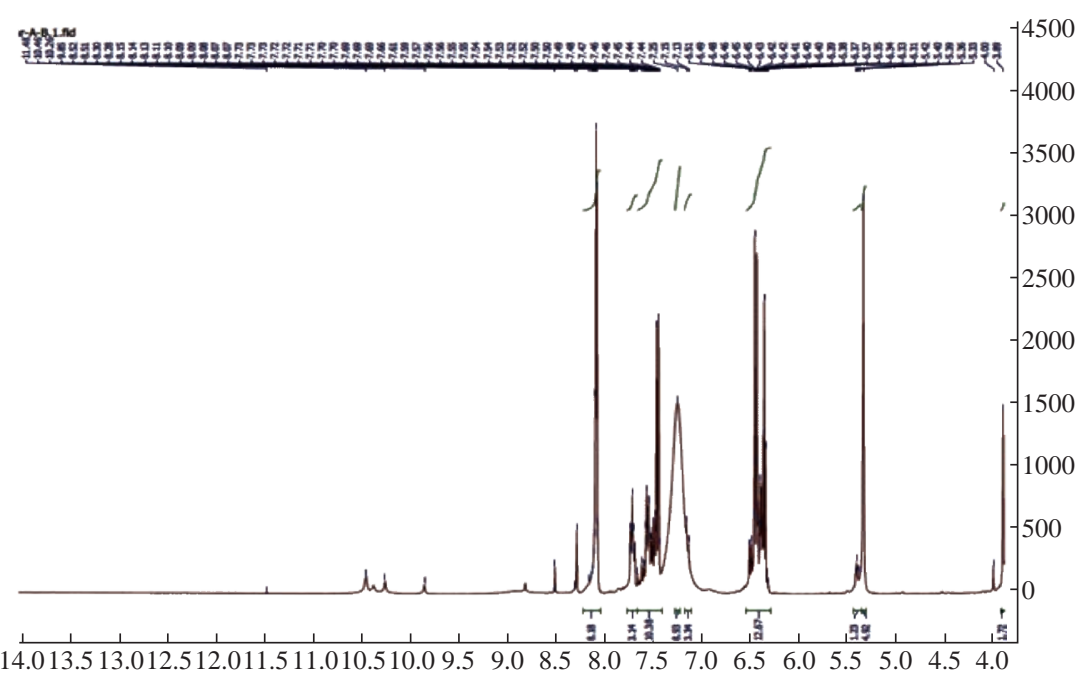

Fig. $10{ }^{1} \mathrm{H}-\mathrm{NMR}$ spectrum of compound [B].

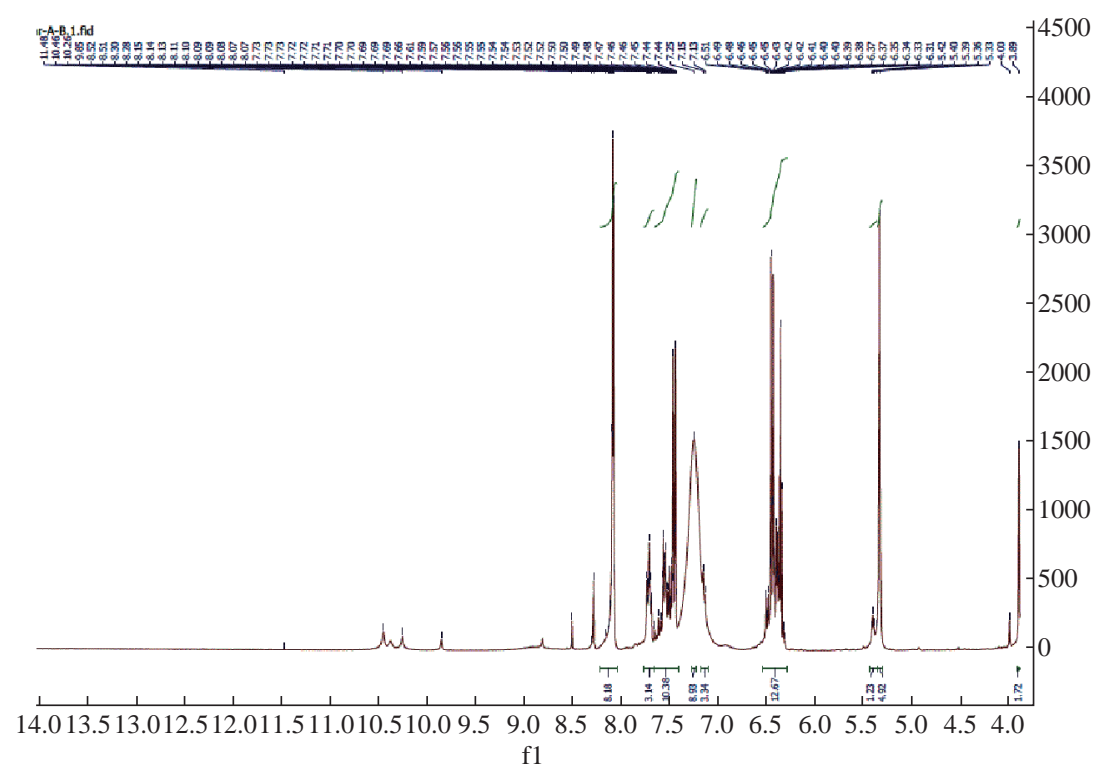

Fig. $11{ }^{1} \mathrm{H}-\mathrm{NMR}$ spectrum of compound [Sh$\left.{ }_{1}\right]$.

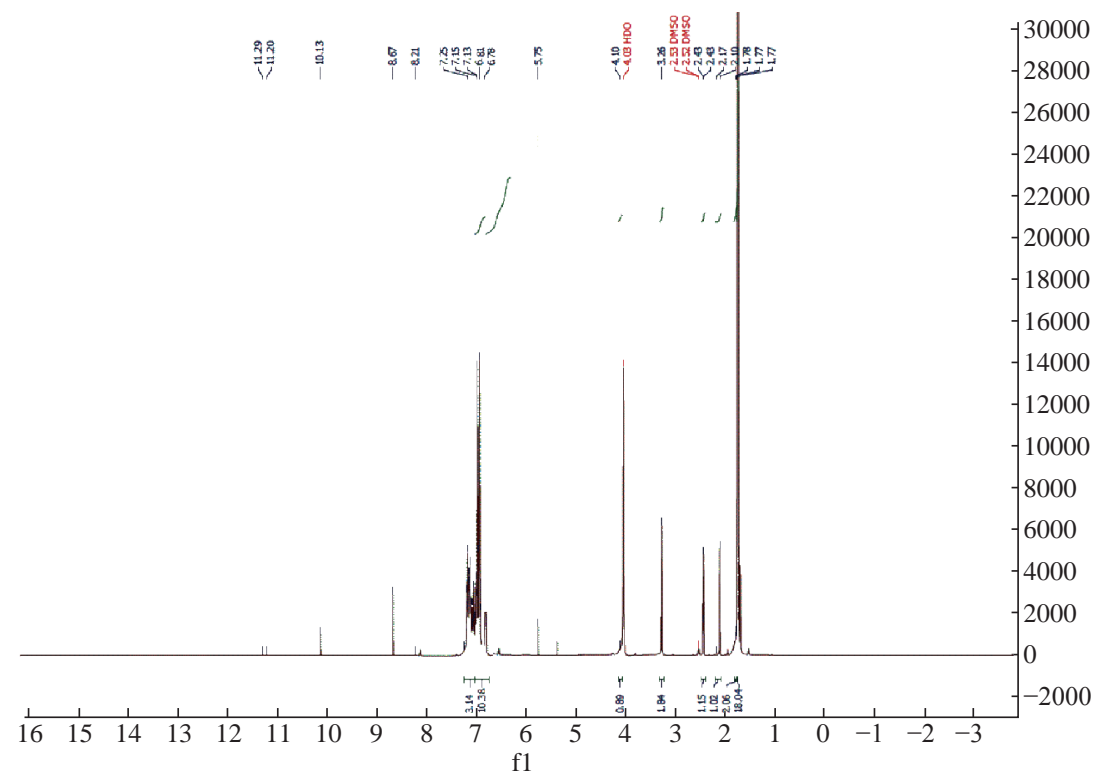

Fig. $12{ }^{1} \mathrm{H}$-NMR spectrum of compound $\left[\mathrm{D}_{1}\right]$. 


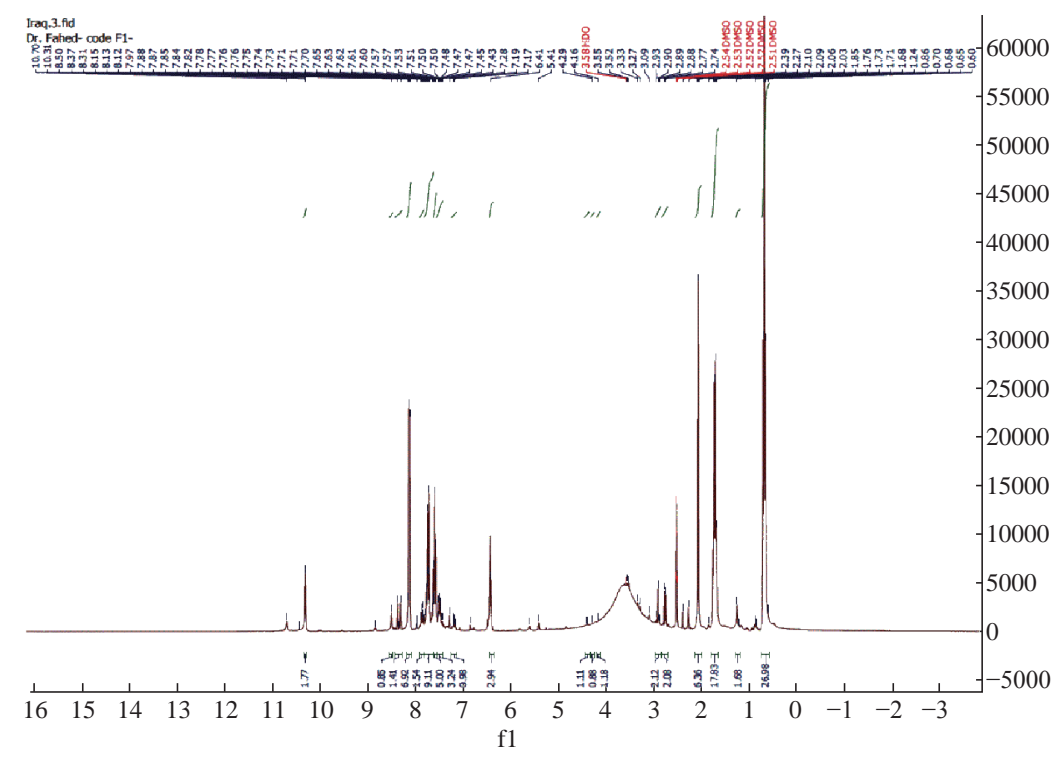

Fig. $13{ }^{1} \mathrm{H}$-NMR spectrum of compound $\left[\mathrm{F}_{1}\right]$.

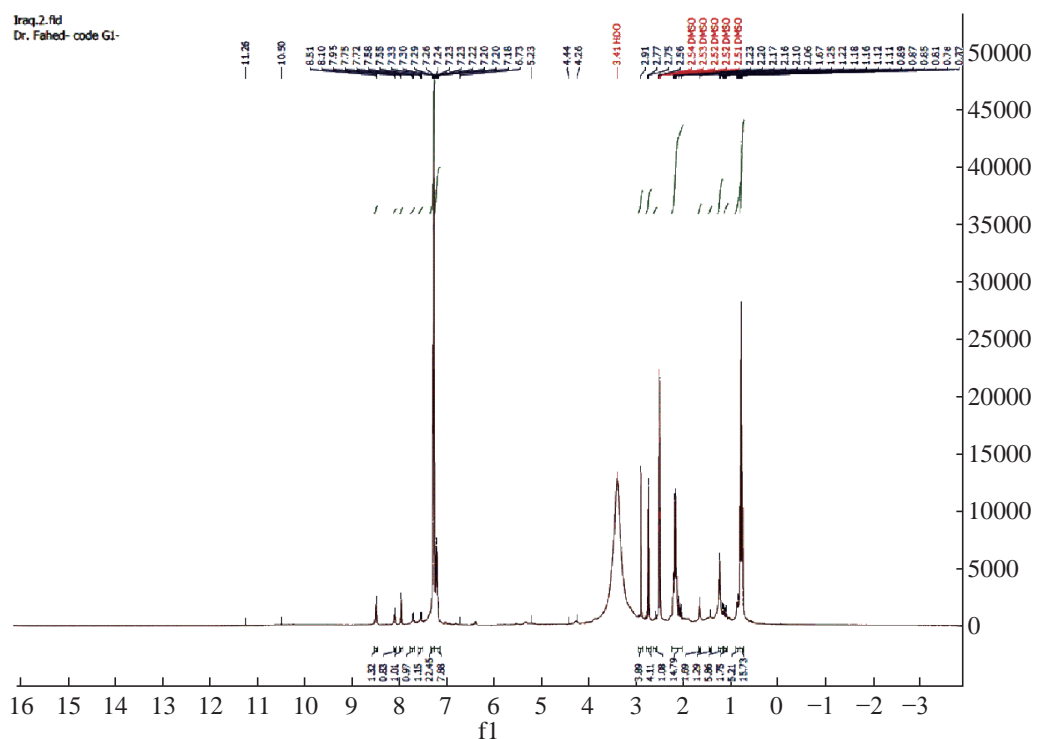

Fig. $14{ }^{1} \mathrm{H}-\mathrm{NMR}$ spectrum of compound $\left[\mathrm{G}_{1}\right]$.

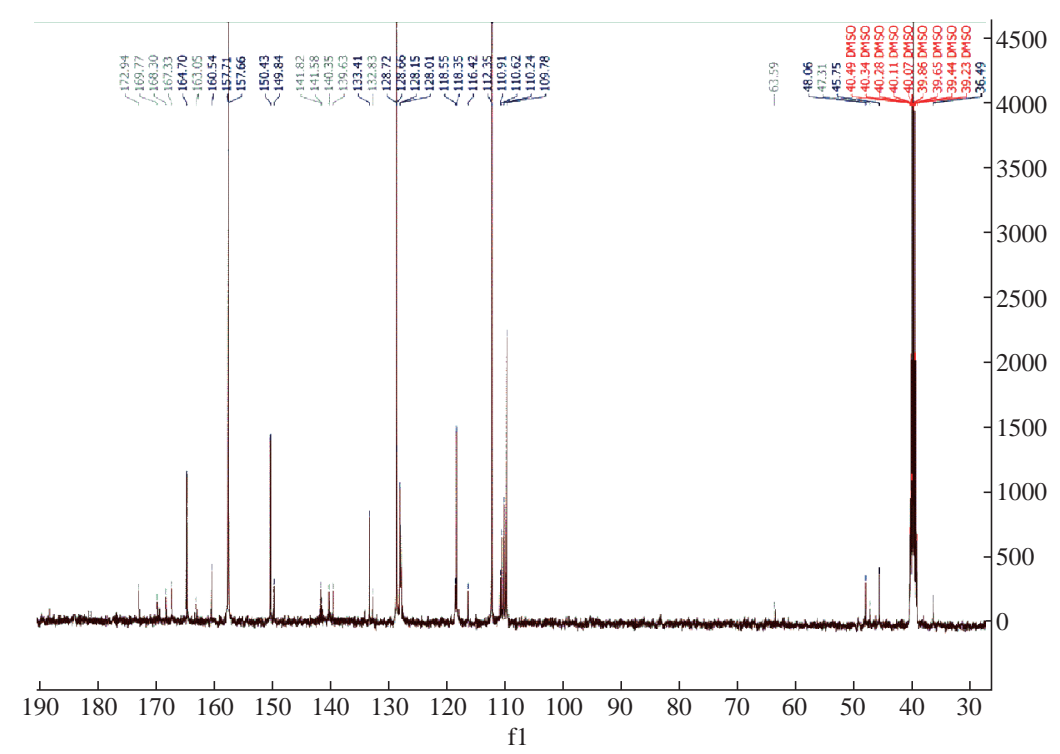

Fig. $15{ }^{13} \mathrm{C}-\mathrm{NMR}$ spectrum of compound [B]. 


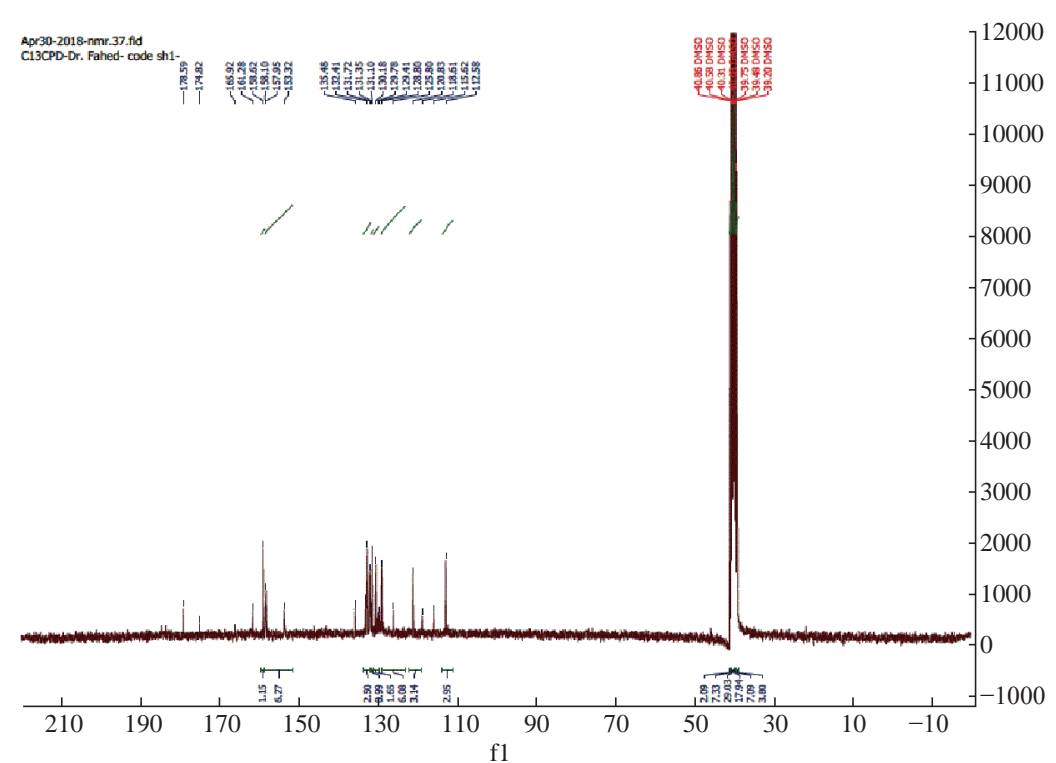

Fig. $16{ }^{13} \mathrm{C}-\mathrm{NMR}$ spectrum of compound $\left[\mathrm{Sh}_{1}\right]$.

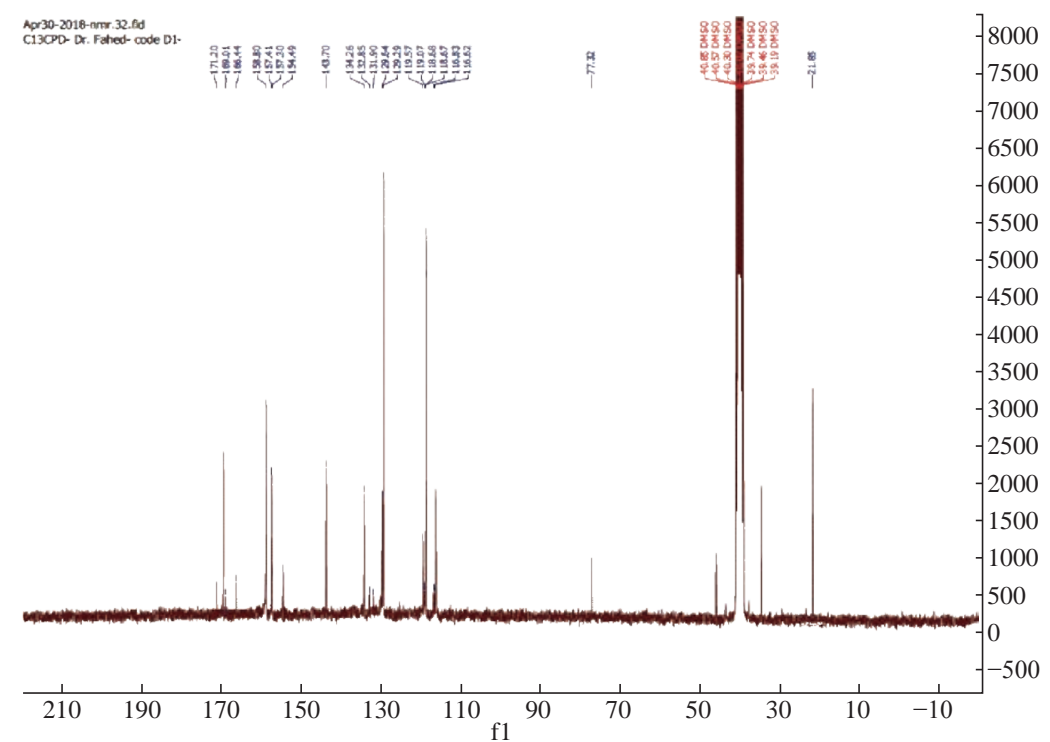

Fig. $17{ }^{13} \mathrm{C}$-NMR spectrum of compound $\left[\mathrm{D}_{1}\right]$.

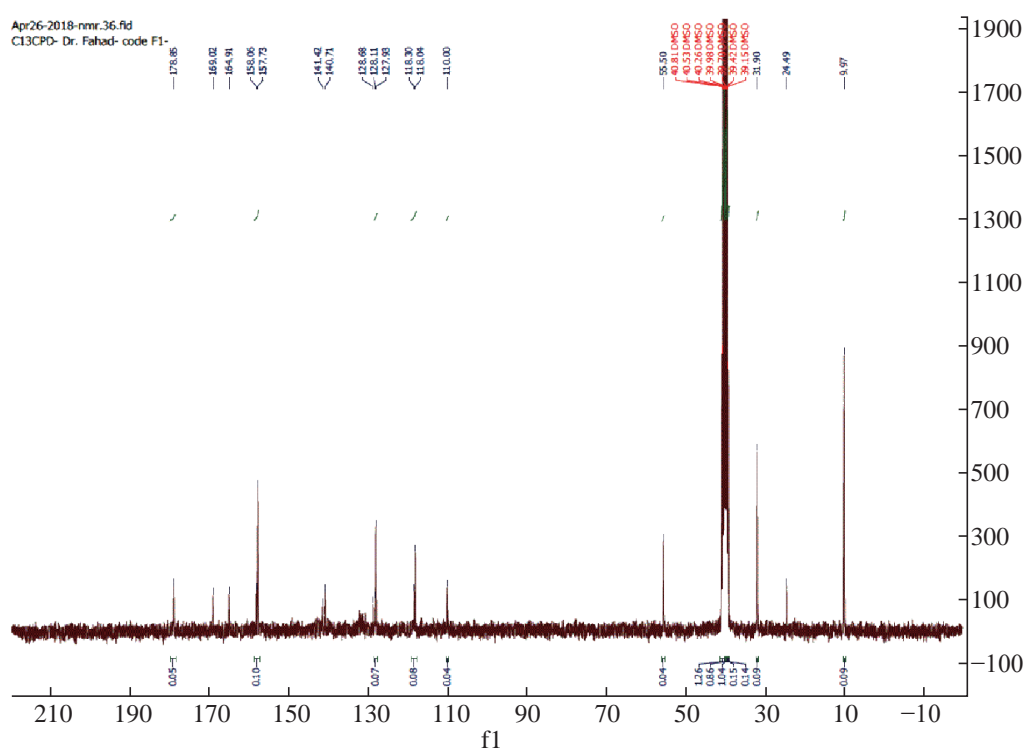

Fig. $18{ }^{13} \mathrm{C}$-NMR spectrum of compound $\left[F_{1}\right]$. 
respectively.

Antimicrobial activity of compounds $\left[\mathrm{F}_{1}\right],\left[\mathrm{G}_{1}\right]$ and $[\mathrm{B}]$ is shown in Fig. 19; antimicrobial activity of compounds $\left[F_{1}\right],\left[G_{1}\right]$ and $[B]$ is shown in Fig. 20.

\section{Conclusions}

In this study, the derivative compounds of barbituric acid from sulfa drugs were stable by resonance with high melting points relatively. They had good antibacterial activity against Pseudomonas aeruginosa and Staphylococcus aureus.

\section{Acknowledgements}

Great thanks to biologist Nadheema Abed Abbas, Department of Ecology, Faculty of Science, the University of Kufa, for his help in the biological test.
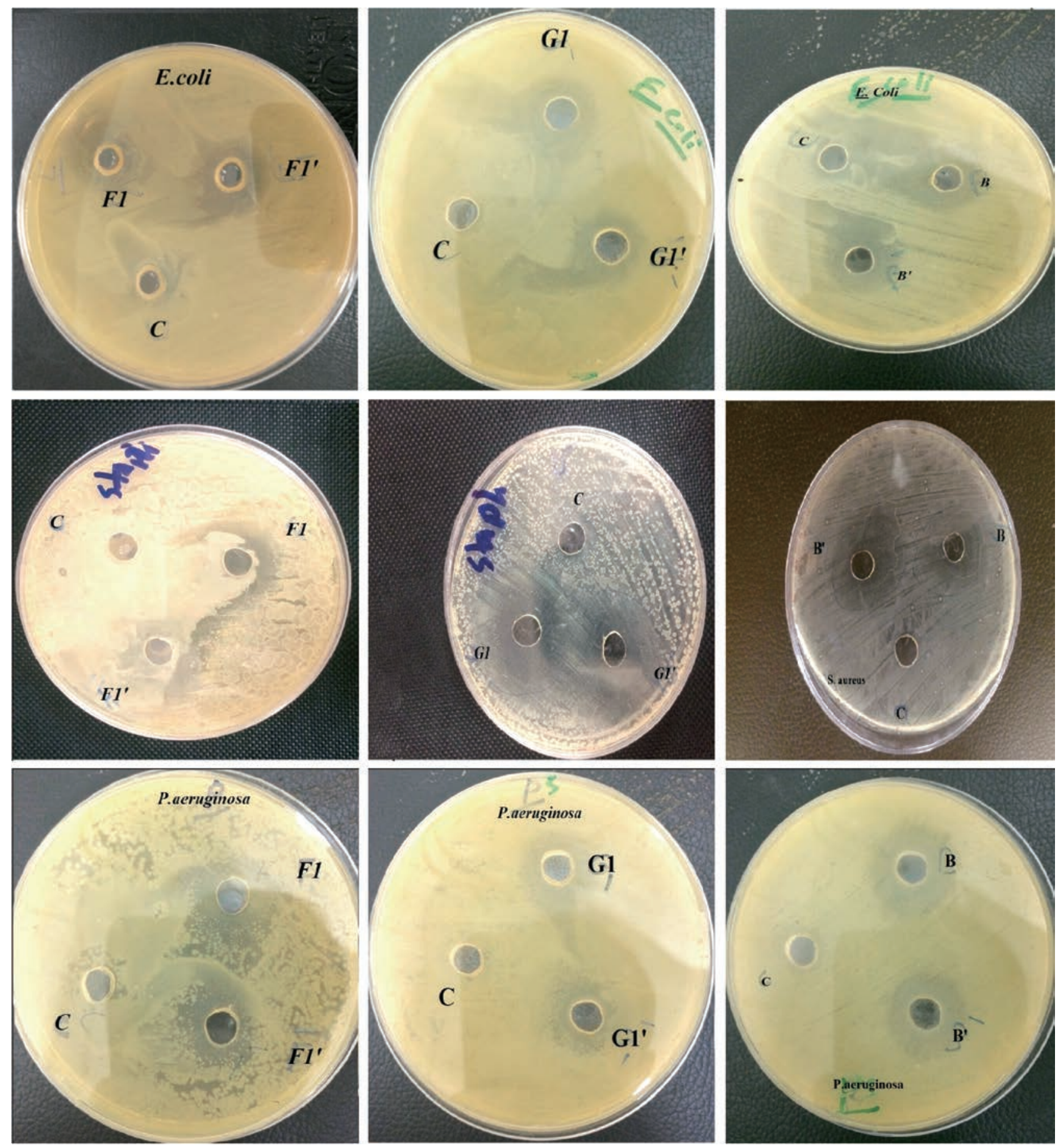

Fig. 19 Antibacterial activity of compounds $\left[\mathrm{F}_{1}\right],\left[\mathrm{G}_{1}\right]$ and $[\mathrm{B}]$. 

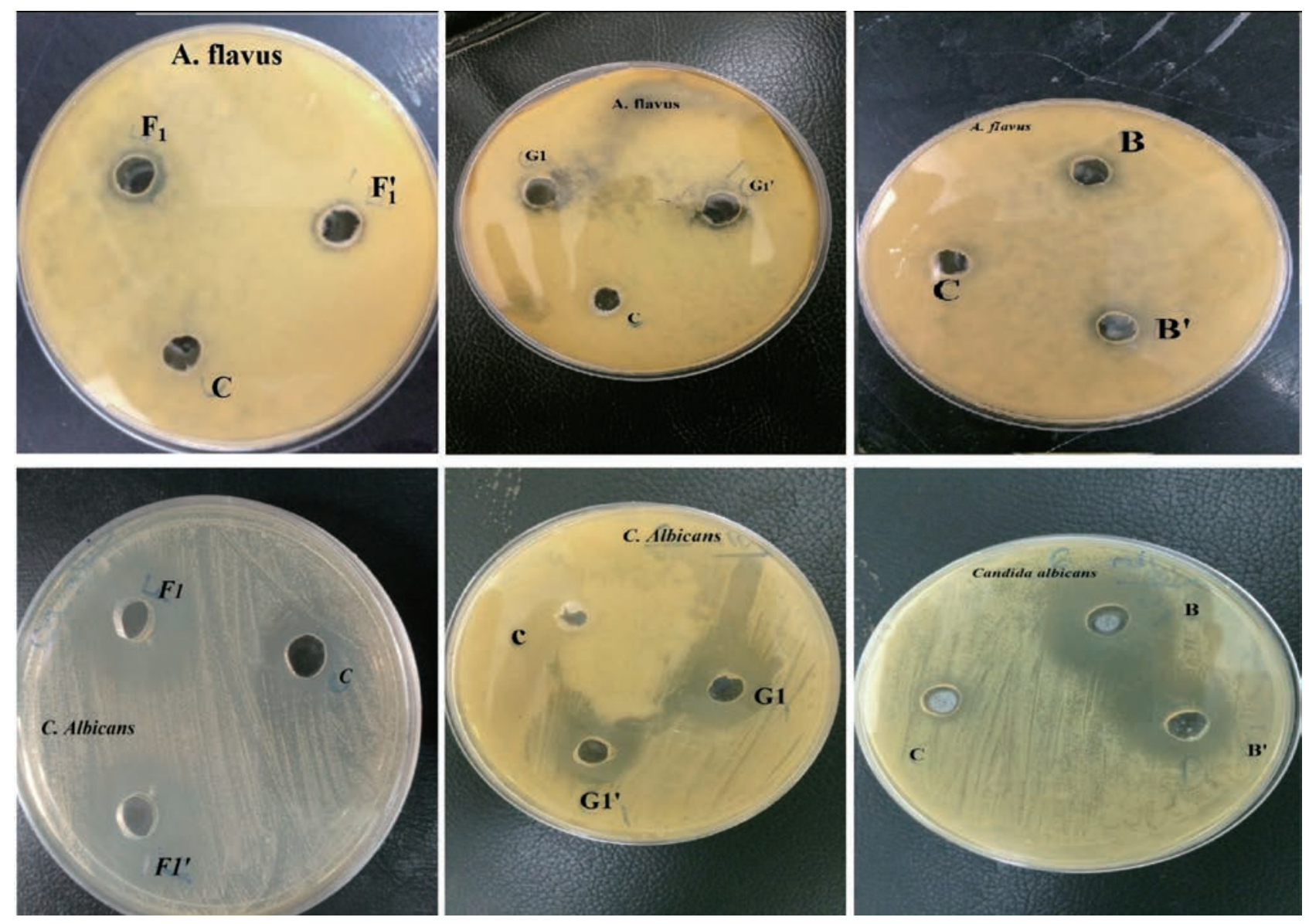

Fig. 20 Antifungal activity of compounds $\left[\mathrm{F}_{1}\right],\left[\mathrm{G}_{1}\right]$ and $[\mathrm{B}]$.

\section{Conflict of Interests}

The authors declare that no competing interest exists.

\section{References}

[1] L. Zhaoliang, H. Zhaohua, S. Lianjun, et al., Synthesis of a new monomer $\mathrm{N}^{\prime}-(\beta$-methacryloyloxy ethyl)-2pyrimidyl-(p-benzyloxy carbonyl) amino benzene sulfonamide and its copolymerization with $\mathrm{N}$-phenyl maleimide. Journal of Polymer Science, 2000, 38(14): 2548-2554.

[2] S.A. Mohammed, H.Y.S. Zebary, Spectrophotometric determination of sulfadiazine via diazotization and coupling reaction- application to pharmaceutical preparations. Raf. J. Sci., 2013, 24(6): 61-73.

[3] V. Kothacota, K.D. Arun, K. Umadevi, et al., Method development and validation of sulphadiazine in bulk and pharmaceutical dosage form by UV-spectrophotometric method. Intern. J. Pharm. Biolog. Arch., 2011, 2(4): 11671171.

[4] A.M. Ghatole, K.R. Lanjewar, and M.K. Gaidhane, Synthesis and anti-microbial activity of some substituted bis[2-((E)-2-(4-benzylideneamino)thiazol-4-yl)-4methylphenol] metal complexes, International Journal of Pharmacy and Pharmaceutical Sciences, 2014, 6(2): 142146.

[5] E. Theophil, H. Siegfried, and S. Andreas, The chemistry of heterocycles (structure, reactions, synthesis, and applications, completely revised and enlarged edition. John Wiley-VCH Verlag \& Co., 2011: 199-200.

[6] G. Vijayta, K. Vinay, A review on biological activity of imidazole and thiazole moieties and their derivatives. Science International, 2013, 1(7): 253-260.

[7] G. Aysegul, T. Mehmet, D. Havva, et al., Cd (II) and $\mathrm{Cu}$ (II) complexes of polydentate Schiff base ligands: synthesis, characterization, properties and biological activity. Inorganica Chimica Acta, 2005, 358(6): 17851797.

[8] S. Siliveru, T. Parthasarathy, Synthesis of sulfonamide based Schiff's bases and their biological evaluation towards Colletorichum gloeosporioides. International Research Journal of Pharmacy, 2012, 3(11): 213-215.

[9] K. Sambasivarao, A.C. Deb, and R.V. Kumar, Spiroannulation of barbituric acid derivatives and its analogs by ring-closing metathesis reaction. Bioorganic \& Medicinal Chemistry Letters, 2005, 15(4): 1039-1043.

[10] M.S. Refat, T. Sharshar, Infrared, Raman, ${ }^{1} \mathrm{H}$ NMR, thermal and positron annihilation lifetime studies of $\mathrm{Pb}(\mathrm{II}), \mathrm{Sn}(\mathrm{II}), \mathrm{Sb}(\mathrm{III}), \mathrm{Bi}(\mathrm{III})-$ barbital complexes. Journal of Molecular Structure, 2012, 1016: 140-146.

[11] A. Andrea, Anharmonic IR and Raman spectra and electronic and vibrational (hyper)polarizabilities of barbituric, 2-thiobarbituric and 2-selenobarbituric acids. Spectrochimica Acta Part A: Molecular and Biomolecular Spectroscopy, 2014, 117: 669-678.

[12] M. Eyad, S. Kamal, E. Jorn, et al., Nucleophilic substitution approach towards 1,3-dimethylbarbituric acid derivatives-new synthetic routes and crystal structures. Tetrahedron, 2012, 68: 1005-1010. 
[13] K.A. Krasnov, P.V. Dorovatovskii, Y.V. Zubavichus, et al., Hydride transfer reactions of 5-(2 -alkohybenzylidene) barbituric acids: Synthesis of 2,4,6-trioxoperhydropyrimidine-5-spiro-30-chromanes. Tetrahedron, 2017, 73(5): 542-549.

[14] A.F. Abbass, E.H. Zimam, Synthesis, characterization and study biological activityof some new pyrimidine and 1, 2, 3, 4-tetrazole derivatives based on sulfadiazine. International Journal of Chem. Tech. Research, 2016, 9(11): 206-217.

[15] N.D. Amnerkar, B.A. Bhongade, and K.P. Bhusari, Synthesis and biological evaluation of some 4-(6-substituted-1,3-benzothiazol-2-yl)amino-1, 3-thiazole-2-amines and their Schiff bases. Arabian Journal of Chemistry, 2015, 8: 545-552.

[16] N.D. Amnerkar, K.P. Bhusari, Synthesis of some thiazolyl aminobenzothiazole derivatives as potential antibacterial, antifungal and anthelmintic agents. Journal of Enzyme Inhibition and Medicinal Chemistry, 2011, 26(1): 22-28.

[17] R.A. Khdur, E.H. Zimam, Synthesis and characterization of some new $\beta$-lactam derivatives from azo sulphadiazine and its biological evaluation as anticancer. Oriental Journal of Chemistry, 2018, 34(1): 371-380.

[18] H. Salman, E.H. Zimam, Synthesis and characterization some novel barbituric acid derivatives from sulfadiazine. Journal of Kufa for Chemical Science, 2012, 6: 86-100.

[19] L. Jacek, Polyhydroxyalkyl derivatives and polyetherols obtained from azacyclic compounds. Polimery, 2011, 56(6): 452-460.

[20] R.M. Mauf, A.H. Sultan, and A.M. Farag, Synthesis of some new 2-(4-aryliminophenoxy) $\mathrm{N}$-arylacetamide via p-hydroxy benzaldehyde. Baghdad Science Journal, 2014, 11(2): 486-490.

Copyright $₫$ Mahmood Muhi Fahad, Ezzat Hussein Zimam, and Majed Jary Mohamad. This is an open-access article distributed under the terms of the Creative Commons Attribution License, which permits unrestricted use, distribution, and reproduction in any medium, provided the original author and source are credited. 